\title{
Representation Results for Law Invariant Time Consistent Functions.*
}

\author{
Michael Kupper ${ }^{\dagger} \quad$ Walter Schachermayer ${ }^{\ddagger}$
}

August 24, 2009

\begin{abstract}
We show that the only dynamic risk measure which is law invariant, time consistent and relevant is the entropic one. Moreover, a real valued function $c$ on $L^{\infty}(a, b)$ is normalized, strictly monotone, continuous, law invariant, time consistent and has the Fatou property if and only if it is of the form $c(X)=u^{-1} \circ \mathbb{E}[u(X)]$, where $u:(a, b) \rightarrow \mathbb{R}$ is a strictly increasing, continuous function. The proofs rely on a discrete version of the Skorohod embedding theorem.
\end{abstract}

Key words: Law invariance, time consistency, certainty equivalent, dynamic risk measures, Skorohod embedding theorem

\section{Introduction and Main Results}

The theory of preferences and their numerical representations goes back to Bernoulli [4]. Axiomatic foundations have been given, among others, by Alt [1], von Neumann and Morgenstern [32], Savage [34], Ellsberg [15], Gilboa and Schmeidler [20] and Maccheroni et al. [30]. If $\succeq$ is a preference order (c.f. Föllmer and Schied [18], Section 2.1) on the set of all probability distributions with bounded support in the interval $(a, b)$, where $-\infty \leq a<b \leq \infty$, such that $\succeq$ satisfies the independence axiom and the Archimedean axiom (c.f. Föllmer and Schied [18], Section 2.1) then it has an affine numerical representation U, which under additional continuity assumptions is a von Neumann-Morgenstern representation (c.f. Föllmer

\footnotetext{
*We thank Freddy Delbaen, Damir Filipović, Hans Föllmer, Fabio Maccheroni, Eberhard Mayerhofer, Alexander Mürmann, Ludger Rüschendorf, Alexander Schied, Mihai Sirbu, Josef Teichmann, Nicolas Vogelpoth and especially Patrick Cheridito as well as two anonymous referees for helpful comments and suggestions.

${ }^{\dagger}$ mkupper@vif.ac.at; Vienna Institute of Finance, University of Vienna and Vienna University of Economics and Business Administration, Heiligenstädter Strasse 46-48, A-1190 Vienna, Austria. Financial support from the Vienna Science and Technology Fund (WWTF) is gratefully acknowledged.

${ }^{\ddagger}$ walter.schachermayer@univie.ac.at; University Vienna, Nordbergstr. 15, 1090 Vienna, Austria. Financial support from the Austrian Science Fund (FWF) under grant P19456, from Vienna Science and Technology Fund (WWTF) under grant MA13 and by the Christian Doppler Research Association (CDG) is gratefully acknowledged.
} 
and Schied [18], Theorem 2.21 and Theorem 2.28). The independence axiom is crucial for a von Neumann-Morgenstern representation. It is demonstrated by Machina [29] and others (see [29] for the references) that preferences which do not satisfy the independence axiom lead to dynamic inconsistencies, at least if the preferences are not updated in an adequate way. In this paper, we show that any numerical representation of a preference order which is defined on the linear space of bounded random variables and which is strictly monotone, normalized on constants, law invariant and time consistent, necessarily is (under some technical continuity conditions, see Theorem 1.4 below) a certainty equivalent of an expected utility.

Here is the formal setting for our results. Let $\left(\Omega, \mathcal{F},\left(\mathcal{F}_{t}\right)_{t \in \mathbb{N}_{0}}, \mathbb{P}\right)$ be a standard filtered probability space, i.e., $(\Omega, \mathcal{F}, \mathbb{P})$ is isomorphic to $[0,1]^{\mathbb{N}_{0}}$ equipped with its Borel sigmaalgebra $\mathcal{B}\left([0,1]^{\mathbb{N}_{0}}\right)$ and the product of Borel measures $\lambda^{\mathbb{N}_{0}}$. The filtration $\left(\mathcal{F}_{t}\right)_{t \in \mathbb{N}_{0}}$ is generated by the coordinate functions. We identify random variables which are a.s. identical. All equalities and inequalities between random variables are understood in the a.s. sense. For $-\infty \leq a<b \leq \infty$, we denote by $L^{\infty}(a, b)=L^{\infty}(\Omega, \mathcal{F}, \mathbb{P} ;(a, b))$ the set of all random variables which are essentially bounded and take values in the open interval $(a, b) . L_{t}^{\infty}(a, b)$ consists of all random variables in $L^{\infty}(a, b)$ which are $\mathcal{F}_{t}$-measurable. Throughout we will simply write $L^{\infty}$ for $L^{\infty}(-\infty, \infty)$ and $L_{t}^{\infty}$ for $L_{t}^{\infty}(-\infty, \infty)$. For $X, Y \in L^{\infty}(a, b)$ we write $X \preceq_{1} Y$ if $Y$ (first order) stochastically dominates $X$, i.e., $\mathbb{P}[X \leq m] \geq \mathbb{P}[Y \leq m]$ for all $m \in \mathbb{R}$.

\subsection{A representation result for law invariant, time consistent certainty equivalents}

On $L^{\infty}(a, b)$ we consider a preference order $\succeq$ with representation $U: L^{\infty}(a, b) \rightarrow \mathbb{R}$ (c.f. Föllmer and Schied [18], Section 2.1) such that $X$ is preferred to $Y$ if $U(X) \geq U(Y)$. We assume that $U$ is law invariant, that is $U(X)=U(Y)$ if $X$ and $Y$ have the same distribution. In the literature, law invariant functions are also referred to as distribution based functions. Thus, the representation $U$ can also be viewed as a function acting on the probability distributions with bounded support in $(a, b)$. We further assume that there exists a certainty equivalent $c_{0}: L^{\infty}(a, b) \rightarrow \mathbb{R}$ for the numerical representation $U$, which is implicitly defined through $U(X)=U\left(c_{0}(X)\right), X \in L^{\infty}(a, b)$. If in addition $U\left(m_{1}\right)>U\left(m_{2}\right)$ for all $m_{1}, m_{2} \in(a, b)$ with $m_{1}>m_{2}$, then the certainty equivalent is normalized on constants, i.e., $c_{0}(m)=m$ for all $m \in(a, b)$. Note that $c_{0}$ is a numerical representation for the preference order $\succeq$ and $c_{0}$ is strictly monotone exactly when $U$ is strictly monotone.

Definition 1.1 A function $c_{0}: L^{\infty}(a, b) \rightarrow \mathbb{R}$ is said to be

- normalized on constants if $c_{0}(m)=m$ for all $m \in(a, b)$;

- strictly monotone if $X \geq Y$ and $\mathbb{P}[X>Y]>0$ imply $c_{0}(X)>c_{0}(Y)$;

- law invariant if $c_{0}(X)=c_{0}(Y)$ whenever law $(X)=\operatorname{law}(Y)$; 
- time consistent if for each $t \in \mathbb{N}_{0}$ there exists a mapping $c_{t}: L^{\infty}(a, b) \rightarrow L_{t}^{\infty}(a, b)$ which satisfies the local property

$$
1_{A} X=1_{A} Y \quad \text { implies } \quad 1_{A} c_{t}(X)=1_{A} c_{t}(Y) \quad \text { for all } A \in \mathcal{F}_{t}, X, Y \in L^{\infty}(a, b)
$$

and

$$
c_{0}(X)=c_{0}\left(c_{t}(X)\right) \text { for all } X \in L^{\infty}(a, b) .
$$

For any function $c_{0}: L^{\infty}(a, b) \rightarrow \mathbb{R}$ which is normalized on constants and strictly monotone, there exists at most one function $c_{t}: L^{\infty}(a, b) \rightarrow L_{t}^{\infty}(a, b)$, which satisfies (1) and (2). If there exists such $c_{t}$, one verifies that it is normalized on constants $\left(c_{t}(m)=m\right.$ for all $\left.m \in L_{t}^{\infty}(a, b)\right)$ and monotone $\left(X \geq Y\right.$ implies $\left.c_{t}(X) \geq c_{t}(Y)\right)$. In view of (2), every time consistent function $c_{0}: L^{\infty}(a, b) \rightarrow L_{t}^{\infty}(a, b)$ restricted to $\bigcup_{t \in \mathbb{N}} L_{t}^{\infty}(a, b)$ can be computed by backward recursion

$$
\left\{\begin{array}{ll}
c_{t}(X)=X & \text { if } t \geq T \\
c_{t}(X)=c_{t}\left(c_{t+1}(X)\right) & \text { if } t<T
\end{array},\right.
$$

where $X \in L_{T}^{\infty}(a, b)$ for some $T \in \mathbb{N}$.

Definition 1.2 ([12, 26]) Let $E$ be a subset of $L^{\infty}$. A function $f: E \rightarrow \mathbb{R}$ has the Fatou property if $f(X) \geq \lim \sup _{n \rightarrow \infty} f\left(X_{n}\right)$ for any $\|\cdot\|_{\infty}$-bounded sequence $X_{n} \in E$ converging to $X \in E$ in probability.

A function $f: E \rightarrow \mathbb{R}$ has the Lebesgue property if $f(X)=\lim _{n \rightarrow \infty} f\left(X_{n}\right)$, for every $\|\cdot\|$-bounded sequence $X_{n} \in E$ converging a.s. to $X \in E$.

Obviously, a function which has the Lebesgue property also has the Fatou property.

Let $b^{\varepsilon, p}$ denote a Bernoulli random variable taking the values $+\varepsilon$ and $-\varepsilon$ with probabilities $p$ and $1-p$.

Definition 1.3 A function $f: L^{\infty}(a, b) \rightarrow \mathbb{R}$ satisfies the continuity condition (C) if for any $\varepsilon>0$ and all $x \in(a, b)$ with $a+\varepsilon<x<b-\varepsilon$ there is $p=p(x) \in(0,1)$ such that

$$
f\left(x+b^{\varepsilon, p}\right)<x .
$$

Note that condition $(\mathrm{C})$ is satisfied if $f$ is strictly monotone and has the Fatou property. Indeed, suppose that $f$ has the Fatou property and let $\left(p_{n}\right)_{n \in \mathbb{N}} \subset(0,1)$ be a sequence with $p_{n} \searrow 0$. Then, $x+b^{\varepsilon, p_{n}} \rightarrow x-\varepsilon$ in probability and the Fatou property and the strict monotonicity of $f$ imply

$$
\limsup _{n \rightarrow \infty} f\left(x+b^{\varepsilon, p_{n}}\right) \leq f(x-\varepsilon)<x .
$$

Hence, there is $p_{n_{0}} \in(0,1)$ with $f\left(x+b^{\varepsilon, p_{n_{0}}}\right)<x$.

Our first main result can now be formulated as follows. 
Theorem 1.4 Let $\left(\Omega, \mathcal{F},\left(\mathcal{F}_{t}\right)_{t \in \mathbb{N}_{0}}, \mathbb{P}\right)$ be a standard filtered probability space and fix numbers $-\infty \leq a<b \leq+\infty$. A function $c_{0}: L^{\infty}(a, b) \rightarrow \mathbb{R}$ is normalized on constants, strictly monotone, $\|\cdot\|_{\infty}$-continuous, law invariant, time consistent and satisfies condition (C) if and only if

$$
c_{0}(X)=u^{-1} \circ \mathbb{E}[u(X)]
$$

for a strictly increasing, continuous function $u:(a, b) \rightarrow \mathbb{R}$. In this case, the function $u$ is uniquely defined up to affine transformations of the form $u \mapsto \alpha u+\beta, \alpha>0, \beta \in \mathbb{R}$, and

$$
c_{t}(X)=u^{-1} \circ \mathbb{E}\left[u(X) \mid \mathcal{F}_{t}\right] \quad \text { for all } t \in \mathbb{N} .
$$

The proof of Theorem 1.4 is postponed to the Section 3 .

Remark 1.5 Theorem 1.4 extends to a dynamic setting the representation results on means by Nagumo [31], Kolmogorov [27] and de Finetti [16]. These representation results give necessary and sufficient conditions for a function $M\left(x_{1}, \ldots, x_{n}\right)$ being a mean, i.e., $M\left(x_{1}, \ldots, x_{n}\right)=\phi^{-1}\left(\sum_{i=1}^{n} \phi\left(x_{i}\right)\right)$ for a continuous, strictly increasing function $\phi$ and any $n \geq 1$ and all values $x_{1}, \ldots, x_{n}$ in an interval $[a, b]$. Hardy et al. [25] give a similar representation result in terms of distribution functions. For further discussions on means, we refer to Hardy et al. [25] and Bullen [5].

Building on the Nagumo-Kolmogorov-de Finetti Theorem, Cerreia-Vioglio et al. [7] recently proved that a function $c_{0}: L^{\infty} \rightarrow \mathbb{R}$ is of the form (5) if and only if $c_{0}$ is normalized on constants, law invariant, monotone, has the Lebesgue property and satisfies

$$
c_{0}\left(X 1_{A}\right)>c_{0}\left(Y 1_{A}\right) \quad \Leftrightarrow \quad c_{0}\left(X 1_{A}+Z 1_{A^{c}}\right)>c_{0}\left(Y 1_{A}+Z 1_{A^{c}}\right)
$$

for all $A \in \mathcal{F}$ and all $X, Y, Z \in L^{\infty}$. Lemma 2 in Carreia-Vioglio et al. [7] further shows that for the case $a=-\infty$ and $b=+\infty$, the function $c_{0}$ is quasi-concave if and only if $u$ is concave.

Remark 1.6 For any continuous and strictly increasing function $u: \mathbb{R} \rightarrow \mathbb{R}$, the functional $\pi(X):=u^{-1} \circ \mathbb{E}[u(X)]$ defines an insurance premium principle, which is called the mean value principle (c.f. Gerber [21], Chapter 5, Section 4). Gerber shows in [22] that any law invariant premium principle $\pi$ which is iterative (i.e. $\pi(X)=\pi(\pi(X \mid Y)$ ) for all $X, Y \in L^{\infty}$, where $\pi(X \mid Y)$ denotes the premium for $X$ given the random variable $\left.Y\right)$ and for which $[0,1] \ni p \mapsto \pi\left(b^{\varepsilon, p}\right)$ is continuous and strictly increasing has to be the mean value principle. Iterativity means that $\pi$ is time consistent for every sub $\sigma$-agebra $\mathcal{G} \subset \mathcal{F}$, i.e., for all $\mathcal{G} \subset \mathcal{F}$ there is $\pi_{\mathcal{G}}: L^{\infty} \rightarrow L^{\infty}(\mathcal{G})$ such that $\pi(X)=\pi\left(\pi_{\mathcal{G}}(X)\right)$ for all $X \in L^{\infty}$ and $\pi_{\mathcal{G}}$ has the local property (1). A premium principle $\pi$ which is time consistent for all sub $\sigma$-agebras $\mathcal{G} \subset \mathcal{F}$ satisfies $(7)$ as $\pi(X)=\pi\left(\pi_{\sigma\left(1_{A}\right)}(X)\right)$ for all $A \in \mathcal{F}$ and all $X \in L^{\infty}$.

It is shown in [21] that $\pi$ is cash invariant (i.e. $\pi(X+m)=\pi(X)+m, m \in \mathbb{R}$ ) if and only if $u$ is an exponential or linear function, see also Nagumo [31] and de Finetti [16]. Cash invariant, time consistent and law invariant functions are discussed in the Subsection 1.2.

Remark 1.7 Suppose that, additionally to the assumptions of Theorem 1.4, we have $c_{0}(X)<\mathbb{E}[X]$ for all $X \in L^{\infty}(a, b)$ unless $X$ is constant. Then $u$ is strictly concave (see for instance Proposition 2.35 in Föllmer and Schied [18]). 


\subsection{A representation result for law invariant, time consistent dynamic risk measures}

In this subsection, we consider functionals which are cash invariant (in the literature this property is also referred to as translation invariance $[2,3])$. This extra condition allows us to prove the main Theorem 1.4 for functions which are not assumed to satisfy condition (C) and are relevant instead of strictly increasing.

Definition 1.8 A dynamic risk measure is a family $\left(\rho_{t}\right)_{t \in \mathbb{N}_{0}}$ of mappings $\rho_{t}: L^{\infty} \rightarrow L_{t}^{\infty}$ such that, for all $X, Y \in L^{\infty}$, the following properties are satisfied

(i) normalization: $\rho_{t}(0)=0$;

(ii) cash invariance: $\rho_{t}(X+m)=\rho_{t}(X)-m$ for all $m \in L_{t}^{\infty}$;

(iii) monotonicity: $X \geq Y$ implies $\rho_{t}(X) \leq \rho_{t}(Y)$.

A dynamic risk measure is

- convex if $\rho_{t}(\lambda X+(1-\lambda) Y) \leq \lambda \rho_{t}(X)+(1-\lambda) \rho_{t}(Y)$ for all $\lambda \in L_{t}^{\infty}$ with $0 \leq \lambda \leq 1$ and all $t \in \mathbb{N}_{0}$;

- law invariant if $\rho_{0}(X)=\rho_{0}(Y)$ whenever law $(X)=\operatorname{law}(Y)$;

- time consistent if $\rho_{0}(X)=\rho_{0}\left(-\rho_{t}(X)\right)$ for all $t \in \mathbb{N}_{0}$;

- relevant if $\rho_{0}\left(-\varepsilon 1_{A}\right)>0$ for all $A \in \mathcal{F}$ and all $\varepsilon>0$.

The theory of risk measures has been initiated by the influential paper by Artzner et al. [2] Since then, risk measures have been generalized in several directions. For an overview of static convex risk measures (mappings $\rho: L^{\infty} \rightarrow \mathbb{R}$ which are normalized, cash invariant, monotone and convex) we refer to Föllmer and Schied [18]. We here are mainly interested in law invariant risk measures which are studied for instance in Kusuoka [28], Frittelli and Rosazza Gianin [19], Jouini et al. [26] and Cheridito and Li [10]. For dynamic risk measures their representations and related concepts such as time consistency, we refer to Artzner et al. [3], Cheridito et al. [8], Cheridito and Kupper [9], Föllmer and Penner [17] and the references therein.

Remark 1.9 It is shown in Jouini et al. [26] that any law invariant convex risk measure $\rho_{0}$ automatically has the Fatou property.

Here is our second main result.

Theorem 1.10 Let $\left(\Omega, \mathcal{F},\left(\mathcal{F}_{t}\right)_{t \in \mathbb{N}_{0}}, \mathbb{P}\right)$ be a standard filtered probability space. The family $\left(\rho_{t}\right)_{t \in \mathbb{N}_{0}}$ is a law invariant, time consistent, relevant dynamic risk measure if and only if there is $\gamma \in(-\infty, \infty]$ such that

$$
\rho_{t}(X)=\frac{1}{\gamma} \ln \mathbb{E}\left[\exp (-\gamma X) \mid \mathcal{F}_{t}\right] \quad \text { for all } t \in \mathbb{N}_{0} .
$$


The limiting cases $\gamma=0$ and $\gamma=\infty$ are defined as

$$
\rho_{t}(X)=\left\{\begin{array}{ll}
\mathbb{E}\left[-X \mid \mathcal{F}_{t}\right] & \gamma=0 \\
{\operatorname{ess} \sup _{Z \in \mathcal{P}_{t}}}\left[Z(-X) \mid \mathcal{F}_{t}\right] & \gamma=\infty
\end{array},\right.
$$

where $\mathcal{P}_{t}$ denotes the set of all positive integrable random variables $Z$ with $\mathbb{E}\left[Z \mid \mathcal{F}_{t}\right]=1$.

In addition, the dynamic risk measure $\left(\rho_{t}\right)_{t \in \mathbb{N}_{0}}$ is convex (resp. coherent) iff $\gamma \in[0, \infty]$ (resp. $\gamma \in\{0, \infty\}$ ).

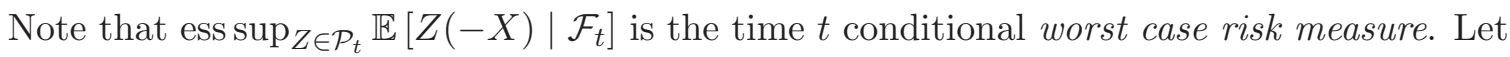
us give some remarks and compare Theorem 1.10 with the existing literature.

Remark 1.11 Due to Corollary 4.59 in Föllmer and Schied [18], any law invariant, convex risk measure is relevant. As a corollary of Theorem 1.10 we deduce that any law invariant, time consistent, convex dynamic risk measure is of the form (8) for some $\gamma \in[0, \infty]$.

Remark 1.12 Closely related to Theorem 1.10 is a result by Freddy Delbaen [13]. In a continuous time framework, under a filtration for which all martingales are continuous, it is shown that the only law invariant, time consistent, dynamic coherent risk measure (a dynamic convex risk measure, which additionally satisfies $\rho_{t}(\lambda X)=\lambda \rho_{t}(X)$ for all $\left.\lambda \in\left(L_{t}^{\infty}\right)_{+}\right)$ is either the negative of the expected value or the worst case risk measure. Independently of the present paper, Delbaen presented at the Oberwolfach meeting (2008) on "Stochastic Analysis in Finance and Insurance" a version of Theorem 1.10 in continuous time, under a filtration generated by a Brownian motion. The result is based on a representation result for dynamic penalty functions by Delbaen, Peng and Rosazza Gianin [14].

Remark 1.13 A continuous time dynamic risk measure can be embedded in our discrete time framework. Indeed, if $\left(\rho_{t}\right)_{t \in[0, T]}$ is a time consistent, dynamic risk measure in continuous time, then for any strictly increasing sequence $0=t_{0}<t_{1}<\ldots, t_{i} \in[0, T]$, the family $\tilde{\rho}_{n}=\rho_{t_{n}}: L^{\infty} \rightarrow L_{t_{n}}^{\infty}$ is a time consistent dynamic risk measure in discrete time. If $\rho_{0}=\tilde{\rho}_{0}$ is law invariant and relevant then Theorem 1.10 states that $\rho_{0}$ has to be the entropic risk measure.

Remark 1.14 Every law invariant, time consistent, dynamic risk measure $\left(\rho_{t}\right)_{t \in \mathbb{N}_{0}}$ is additive for independent random variables, i.e.,

$$
\rho_{0}(X+Y)=\rho_{0}(X)+\rho_{0}(Y) \text { for all } X, Y \in L^{\infty} \text { being independent. }
$$

In Goovaerts et al. [24] it is shown that any risk measure satisfying (10) is a weighted average of entropic risk measures:

$$
\begin{aligned}
-\rho_{0}(X)= & G(-\infty) \operatorname{ess} \cdot \inf X+\int_{(-\infty, \infty)}-\frac{1}{\gamma} \ln \mathbb{E}[\exp (-\gamma X)] d G(\gamma) \\
& +(1-(G(\infty)) \operatorname{ess} \cdot \sup X
\end{aligned}
$$


for an increasing function $G:[-\infty, \infty] \rightarrow[0,1]$. Related results for premium principles satisfying a different monotonicity assumption have axiomatically been characterized by Gerber and Goovaerts [23].

We finally sketch why (10) follows from the above assumptions. Indeed, there exist $X^{\prime} \in L_{1}^{\infty}$ and $Y^{\prime} \in L_{2}^{\infty}$ such that $Y^{\prime}$ is independent of $\mathcal{F}_{1}$, law $\left(X^{\prime}\right)=\operatorname{law}(X)$ and $\operatorname{law}\left(Y^{\prime}\right)=$ law $(Y)$. By Lemma 2.2 below, it follows

$$
\rho_{0}(X+Y)=\rho_{0}\left(X^{\prime}+Y^{\prime}\right)=\rho_{0}\left(X^{\prime}-\rho_{1}\left(Y^{\prime}\right)\right)=\rho_{0}(X)+\rho_{0}(Y) .
$$

Remark 1.15 Weber [35] studies law invariant dynamic convex risk measures which satisfy a weaker time consistency property. More precisely, he shows that if $\rho_{t}$ is weakly acceptance and rejection consistent then $\rho_{0}$ has to be a shortfall risk measure.

Remark 1.16 Artzner et al. [3] and Cheridito and Stadje [11] provide explicit counterexamples which demonstrate that the average value at risk $A V @ R$ and the value at risk $V @ R$ are not time consistent. Note that both risk measures are law invariant, but $V @ R$ even fails to be convex.

\section{Proof of Theorem 1.10}

Throughout this section, $\left(\Omega, \mathcal{F},\left(\mathcal{F}_{t}\right)_{t \in \mathbb{N}_{0}}, \mathbb{P}\right)$ is a standard filtered probability space.

Proof of the "if"-part of Theorem 1.4.

It is straightforward to check that any dynamic risk measure $\left(\rho_{t}\right)_{t \in \mathbb{N}_{0}}$ of the form (8) defines a law invariant, time consistent, relevant dynamic risk measure.

Proof of the "only if"-part of Theorem 1.4.

Let $\left(\rho_{t}\right)_{t \in \mathbb{N}_{0}}$ be a law invariant, time consistent, relevant dynamic risk measure. Let us define the collection of utility functions

$$
u_{\gamma}(x)=\left\{\begin{array}{ll}
\frac{1-\exp (-\gamma x)}{1-\exp (-\gamma)} & \text { if } \gamma \in \mathbb{R} \backslash\{0\} \\
x & \text { if } \gamma=0
\end{array},\right.
$$

satisfying $u_{\gamma}(0)=0, u_{\gamma}(1)=1$ and $u_{\gamma}: \mathbb{R} \rightarrow \mathbb{R}$ is increasing, for all $\gamma \in \mathbb{R}$. For every sequence $\left(\gamma_{k}\right)_{k \in \mathbb{N}} \subset \mathbb{R}$ with $\gamma_{k} \rightarrow \gamma \in \mathbb{R}$, the sequence $u_{\gamma_{k}}$ converges uniformly on compacts to $u_{\gamma}$. The entropic risk measure with risk aversion parameter $\gamma \in \mathbb{R}$ is defined by

$$
\rho^{\gamma}(X)=\left\{\begin{array}{ll}
\frac{1}{\gamma} \ln \mathbb{E}[\exp (-\gamma X)] & \text { if } \gamma \in \mathbb{R} \backslash\{0\} \\
\mathbb{E}[-X] & \text { if } \gamma=0
\end{array}, \quad X \in L^{\infty}\right.
$$

Note that $\rho^{\gamma}(X)=-u_{\gamma}^{-1} \circ \mathbb{E}\left[u_{\gamma}(X)\right]$ for all $X \in L^{\infty}$.

Lemma 2.1 The collection of entropic risk measures $\rho^{\gamma}, \gamma \in \mathbb{R}$, satisfies for all $X \in L^{\infty}$

(i) $\lim _{\gamma \rightarrow \infty} \rho^{\gamma}(X)=\sup _{Z \in \mathcal{P}_{0}} \mathbb{E}[Z(-X)]=\operatorname{ess} \cdot \sup (-X)$, 
(ii) $\lim _{\gamma \rightarrow 0} \rho^{\gamma}(X)=\mathbb{E}[-X]$,

(iii) $\lim _{\gamma \rightarrow-\infty} \rho^{\gamma}(X)=\inf _{Z \in \mathcal{P}_{0}} \mathbb{E}[Z(-X)]=\operatorname{ess} . \inf (-\mathrm{X})$ and

(iv) the function $\mathbb{R} \ni \gamma \mapsto \rho^{\gamma}(X)$ is increasing.

The set $\mathcal{P}_{0}$ consists of all probability densities, i.e., all positive integrable random variables $Z$ with $\mathbb{E}[Z]=1$.

Proof. (i) follows from the well-known dual representation for the entropic risk measure (see for instance in $[8,17,18]$ )

$$
\rho^{\gamma}(X)=\sup _{Z \in \mathcal{P}_{0}}\left\{\mathbb{E}[Z(-X)]-\frac{1}{\gamma} \mathbb{E}[Z \ln Z]\right\}, \quad X \in L^{\infty}, \gamma>0
$$

Equation (11) further yields

$$
\lim _{\gamma \searrow 0} \rho^{\gamma}(X)=\mathbb{E}[-X] \text { and } \quad \mathbb{R}_{+} \ni \gamma \mapsto \rho^{\gamma}(X) \text { is increasing. }
$$

(ii), (iii) and (iv) then follow from (i), (12) and the equality $\rho^{\gamma}(X)=-\rho^{-\gamma}(-X)$ valid for all $X \in L^{\infty}$ and all $\gamma \in \mathbb{R}$.

Lemma 2.1 justifies the definition (9). Let $\rho^{\infty}\left(\rho^{-\infty}\right)$ be defined as the worst (best) case risk measure. Let $b_{1}, b_{2}, b_{3}, \ldots$ denote a sequence of independent Bernoulli random variables, such that $b_{t}$ is independent of $\mathcal{F}_{t-1}$ and assumes the values 1 and -1 with probabilities $1 / 2$. Define

$$
\eta_{\varepsilon}:=\rho_{0}\left(\varepsilon b_{1}\right) \quad \text { implying } \quad \rho_{0}\left(\varepsilon b_{1}+\eta_{\varepsilon}\right)=0 .
$$

Clearly, $-\varepsilon \leq \eta_{\varepsilon} \leq \varepsilon$. For instance, if $\rho_{0}$ is the worst case risk measure, then $\eta_{\varepsilon}=\varepsilon$. Define $\gamma_{\varepsilon} \in[-\infty, \infty]$ implicitly through $\rho^{\gamma_{\varepsilon}}\left(\varepsilon b_{1}+\eta_{\varepsilon}\right)=0$. In particular, if $\gamma_{\varepsilon} \in \mathbb{R}$, then

$$
u_{\gamma_{\varepsilon}}(x)=\mathbb{E}\left[u_{\gamma_{\varepsilon}}\left(x+\varepsilon b_{1}+\eta_{\varepsilon}\right)\right], \quad \text { for all } x \in \mathbb{R} .
$$

The goal is to show that there exists $\gamma \in(-\infty, \infty]$ such that

$$
\rho_{0}(X)=\rho^{\gamma}(X) \text { for all } X \in L^{\infty},
$$

which in turn implies (8).

Lemma 2.2 Let $t \in \mathbb{N}$ and $X, Y \in L^{\infty}$ with $\operatorname{law}(X)=\operatorname{law}(Y)$ and $Y$ is independent of $\mathcal{F}_{t}$. Then, we have $\rho_{0}(X)=\rho_{t}(Y)$.

Proof. Suppose that $\rho_{t}(Y)$ is not constant. Then, there exist $m \in \mathbb{R}$ and $A, A^{\prime} \in \mathcal{F}_{t}$ with $\mathbb{P}[A]=\mathbb{P}\left[A^{\prime}\right]>0$ such that $\rho_{t}(Y)-m>0$ on $A$ and $\rho_{t}(Y)-m \leq 0$ on $A^{\prime}$. By time consistency, local property and cash invariance of $\rho_{t}$, we deduce

$$
\rho_{0}\left(1_{A}\left(m-\rho_{t}(Y)\right)=\rho_{0}\left(1_{A}\left(-\rho_{t}(Y+m)\right)=\rho_{0}\left(-\rho_{t}\left(1_{A}(Y+m)\right)\right)=\rho_{0}\left(1_{A}(Y+m)\right)\right.\right.
$$


and analogously

$$
\rho_{0}\left(1_{A^{\prime}}\left(m-\rho_{t}(Y)\right)=\rho_{0}\left(1_{A^{\prime}}(Y+m)\right) .\right.
$$

On the one hand, since $\rho_{0}$ is relevant, it follows

$$
\rho_{0}\left(1_{A}\left(m-\rho_{t}(Y)\right)\right)>0 \geq \rho_{0}\left(1_{A^{\prime}}\left(m-\rho_{t}(Y)\right)\right) .
$$

On the other hand, law $\left(1_{A}(Y+m)\right)=\operatorname{law}\left(1_{A^{\prime}}(Y+m)\right)$ implying that $\rho_{0}\left(1_{A}(Y+m)\right)=$ $\rho_{0}\left(1_{A^{\prime}}(Y+m)\right)$ in contradiction to $(16),(17)$ and (18). Hence, $\rho_{t}(Y)$ has to be constant. Then, since law $(X)=\operatorname{law}(Y)$, we get

$$
\rho_{0}(X)=\rho_{0}(Y)=\rho_{0}\left(-\rho_{t}(Y)\right)=\rho_{t}(Y)
$$

by time consistency and cash invariance of $\rho_{0}$. This completes the proof.

For any $\varepsilon>0$, we define the random walk with drift

$$
R_{t}^{\varepsilon}:=R_{0}^{\varepsilon}+\sum_{j=1}^{t}\left(\varepsilon b_{j}+\eta_{\varepsilon}\right), \quad t \in \mathbb{N}_{0},
$$

starting at $R_{0}^{\varepsilon} \in \mathbb{R}$. The following Lemma shows that any $R^{\varepsilon}$ of the form (19) satisfies $\rho_{t}\left(R_{s}^{\varepsilon}\right)=-R_{t}^{\varepsilon}$ for all $s \geq t$, which can be viewed as a generalized martingale property with respect to the non-linear conditional expectation $\rho_{t}$.

Lemma 2.3 Let $R^{\varepsilon}$ be a stochastic process which follows the dynamics (19) and let $\tau$ be a bounded stopping time. Then, we have $\rho_{0}\left(R_{\tau}^{\varepsilon}\right)=-R_{0}^{\varepsilon}$.

Proof. We first show that

$$
\rho_{s}\left(R_{s+1}^{\varepsilon}\right)=-R_{s}^{\varepsilon} \text { for all } s \in \mathbb{N}_{0} \text {. }
$$

Indeed, suppose that $R_{s}^{\varepsilon}$ assumes the values $\left\{x_{1}, \ldots, x_{N}\right\}$, i.e., $\mathbb{P}\left[R_{s}^{\varepsilon} \in\left\{x_{1}, \ldots, x_{N}\right\}\right]=1$. In view of (19) we have

$$
R_{s+1}^{\varepsilon}=\sum_{n=1}^{N} 1_{\left\{R_{s}^{\varepsilon}=x_{n}\right\}}\left(x_{n}+\varepsilon b_{s+1}+\eta_{\varepsilon}\right) .
$$

Hence, the local property of $\rho_{s}$ yields

$$
\rho_{s}\left(R_{s+1}^{\varepsilon}\right)=\sum_{n=1}^{N} 1_{\left\{R_{s}^{\varepsilon}=x_{n}\right\}} \rho_{s}\left(x_{n}+\varepsilon b_{s+1}+\eta_{\varepsilon}\right) .
$$

Since law $\left(x_{n}+\varepsilon b_{s+1}+\eta_{\varepsilon}\right)=\operatorname{law}\left(x_{n}+\varepsilon b_{1}+\eta_{\varepsilon}\right)$ a.s., and $x_{n}+\varepsilon b_{s+1}+\eta_{\varepsilon}$ is independent of $\mathcal{F}_{s}$, Lemma 2.2 and (13) imply

$$
\rho_{s}\left(x_{n}+\varepsilon b_{s+1}+\eta_{\varepsilon}\right)=\rho_{0}\left(x_{n}+\varepsilon b_{1}+\eta_{\varepsilon}\right)=-x_{n} .
$$


We then derive (20) from (21) and (22).

We next show by backward induction that

$$
\rho_{t}\left(R_{\tau}^{\varepsilon}\right)=-R_{t \wedge \tau}^{\varepsilon} \text { for all } t \in \mathbb{N}_{0} .
$$

Indeed, since $\tau$ is a bounded stopping time there is $T \in \mathbb{N}$ with $\tau \leq T$. By cash invariance of $\rho_{T}$ we have $\rho_{T}\left(R_{\tau}^{\varepsilon}\right)=-R_{\tau}^{\varepsilon}=-R_{T \wedge \tau}^{\varepsilon}$. For the induction step, we assume that (23) holds for all $t \geq s+1$. In view of (20) we deduce on $A:=\{\tau \geq s+1\} \in \mathcal{F}_{s}$

$$
1_{A} \rho_{s}\left(R_{\tau}^{\varepsilon}\right)=1_{A} \rho_{s}\left(-\rho_{s+1}\left(R_{\tau}^{\varepsilon}\right)\right)=1_{A} \rho_{s}\left(R_{(s+1) \wedge \tau}^{\varepsilon}\right)=1_{A} \rho_{s}\left(R_{s+1}^{\varepsilon}\right)=-1_{A} R_{s \wedge \tau}^{\varepsilon} .
$$

By cash invariance of $\rho_{s}$, we deduce on $A^{c}=\{\tau \leq s\}$

$$
1_{A^{c}} \rho_{s}\left(R_{\tau}^{\varepsilon}\right)=1_{A^{c}} \rho_{s}\left(-\rho_{s+1}\left(R_{\tau}^{\varepsilon}\right)\right)=1_{A^{c}} \rho_{s}\left(R_{(s+1) \wedge \tau}^{\varepsilon}\right)=1_{A^{c}} \rho_{s}\left(R_{s \wedge \tau}^{\varepsilon}\right)=-1_{A^{c}} R_{s \wedge \tau}^{\varepsilon} .
$$

Combining (24) with (25) implies $\rho_{s}\left(R_{\tau}^{\varepsilon}\right)=-R_{s \wedge \tau}^{\varepsilon}$ and the induction step is completed.

Remark 2.4 For any $R^{\varepsilon}$ of the form (19) with respective $\gamma_{\varepsilon} \in \mathbb{R}$, the stochastic process $u_{\gamma_{\varepsilon}}\left(R_{t}^{\varepsilon}\right)$ is a martingale. Indeed, for all $t \in \mathbb{N}_{0}$ we deduce from (14) that

$$
\mathbb{E}\left[u_{\gamma_{\varepsilon}}\left(R_{t+1}^{\varepsilon}\right) \mid \mathcal{F}_{t}\right]=\mathbb{E}\left[u_{\gamma_{\varepsilon}}\left(R_{t}^{\varepsilon}+\varepsilon b_{t+1}+\eta_{\varepsilon}\right) \mid \mathcal{F}_{t}\right]=u_{\gamma_{\varepsilon}}\left(R_{t}^{\varepsilon}\right) .
$$

The proof is based on the following discrete version of the Skorohod embedding theorem (see for instance Revuz and Yor [33], chapter VI, §5, and the references therein).

Lemma 2.5 Let $X \in L^{\infty}$ and $\left(\varepsilon_{k}\right)_{k \in \mathbb{N}}$ be a sequence tending to zero such that $\gamma_{k}:=\gamma_{\varepsilon_{k}} \in \mathbb{R}$ and $\left(\gamma_{k}\right)_{k \in \mathbb{N}}$ converges to some $\gamma \in \mathbb{R}$ as $k$ tends to infinity. Then, there exists a subsequence of $\left(\varepsilon_{k}\right)$ (still denoted by $\left(\varepsilon_{k}\right)$ ), such that for any $k \in \mathbb{N}$ we may find stochastic processes $R^{\varepsilon_{k},+}, R^{\varepsilon_{k},-}$ of the form (19) as well as bounded stopping times $\sigma_{k}^{+}$and $\sigma_{k}^{-}$, such that $X$ satisfies

$$
R_{\sigma_{k}^{+}}^{\varepsilon_{k},+} \succeq_{1} X \succeq_{1} R_{\sigma_{k}^{-}}^{\varepsilon_{k},-}
$$

and

$$
\lim _{k \rightarrow \infty}\left|u_{\gamma_{k}}\left(R_{0}^{\varepsilon_{k},+}\right)-\mathbb{E}\left[u_{\gamma_{k}}(X)\right]\right|=\lim _{k \rightarrow \infty}\left|u_{\gamma_{k}}\left(R_{0}^{\varepsilon_{k},-}\right)-\mathbb{E}\left[u_{\gamma_{k}}(X)\right]\right|=0 .
$$

Proof. A discrete version of the Skorohod embedding theorem is the heart of the construction. The technical details are a little messy but the basic idea is straightforward. For the convenience of the reader we first informally sketch the idea. The process $R^{\varepsilon_{k}}$ is a random walk with drift. Further, $u_{\gamma_{k}}\left(R^{\varepsilon_{k}}\right)$ is a martingale. We approximate a given $X \in L^{\infty}$ in law by the terminal value $R_{\sigma}^{\varepsilon_{k}}$, where $\sigma$ is a bounded stopping time. The goal is to have $u_{\varepsilon_{k}}\left(R_{0}^{\varepsilon_{k}}\right)=\mathbb{E}\left[u_{\varepsilon_{k}}\left(R_{\sigma}^{\varepsilon_{k}}\right)\right] \approx \mathbb{E}\left[u_{\varepsilon_{k}}(X)\right]$. We first assume that $X$ only assumes two values $x_{1}<x_{2}$. We start the random walk $R^{\varepsilon_{k}}$ at $R_{0}^{\varepsilon_{k}}=u_{\gamma_{k}}^{-1} \circ \mathbb{E}\left[u_{\gamma_{k}}(X)\right] \in\left(x_{1}, x_{2}\right)$ and define the stopping time $\sigma=\inf \left\{t \in \mathbb{N}_{0} \mid R_{t}^{\varepsilon_{k}} \approx x_{1}\right.$ or $\left.R_{t}^{\varepsilon_{k}} \approx x_{2}\right\}$. By martingale convergence, $\sigma$ is a.s. finite and $\mathbb{P}\left[R_{\sigma}^{\varepsilon_{k}} \approx x_{1}\right] \approx \mathbb{P}\left[X=x_{1}\right]$ and $\mathbb{P}\left[R_{\sigma}^{\varepsilon_{k}} \approx x_{2}\right] \approx \mathbb{P}\left[X=x_{2}\right]$. We then need some technicalities to make the meaning of " $\approx$ " precise and to replace $\sigma$ by a bounded stopping time. We then repeat the above argument along a binomial tree. 
We now state the proof in full detail. It is enough to prove the lemma for random variables $X$ which assume $2^{N}$ different values for some $N \in \mathbb{N}$. Indeed, approximate $X \in L^{\infty}$ by some $X^{N} \in L^{\infty}$ which takes $2^{N}$ different values such that $\left\|X-X^{N}\right\|_{\infty} \leq 1 / N$ and

$$
X^{N}+1 / N \succeq_{1} X \succeq_{1} X^{N}-1 / N .
$$

Applying the lemma (valid for random variables $X$ which assume $2^{N}$ values) on $X^{N}+1 / N$ and $X^{N}-1 / N$ yields stochastic processes $R^{\varepsilon_{k}, N,+}, R^{\varepsilon_{k}, N,-}$ and bounded stopping times $\sigma_{k, N}^{+}$and $\sigma_{k, N}^{-}$such that

$$
\begin{gathered}
R_{\sigma_{k, N}^{+}}^{\varepsilon_{k}, N,+} \succeq_{1} X^{N}+1 / N \succeq_{1} X \succeq_{1} X^{N}-1 / N \succeq_{1} R_{\sigma_{k, N}^{-}}^{\varepsilon_{k}, N,-} \\
\lim _{k \rightarrow \infty}\left|u_{\gamma_{k}}\left(R_{0}^{\varepsilon_{k}, N,+}\right)-\mathbb{E}\left[u_{\gamma_{k}}\left(X^{N}+1 / N\right)\right]\right|=\lim _{k \rightarrow \infty}\left|u_{\gamma_{k}}\left(R_{0}^{\varepsilon_{k}, N,-}\right)-\mathbb{E}\left[u_{\gamma_{k}}\left(X^{N}-1 / N\right)\right]\right|=0 .
\end{gathered}
$$

The claim then follows since for any $k \in \mathbb{N}$ there is $N=N(k)$ such that

$$
\left|\mathbb{E}\left[u_{\gamma_{k}}\left(X^{N}+1 / N\right)\right]-\mathbb{E}\left[u_{\gamma_{k}}(X)\right]\right| \leq 1 / k .
$$

Suppose now that $X$ takes the values $x_{1}<\cdots<x_{2^{N}}$ with respective probabilities $p_{1}, \ldots, p_{2^{N}}$. Let us introduce the finite probability space

$$
\left(\hat{\Omega}=\left\{\omega_{1}, \ldots, \omega_{2^{N}}\right\}, \hat{\mathcal{F}}=2^{\hat{\Omega}}, \hat{\mathbb{P}}=\left\{p_{1}, \ldots, p_{2^{N}}\right\}\right) .
$$

$\hat{\mathbb{E}}$ denotes the (conditional) expectation with respect to $\hat{\mathbb{P}}$. The filtration $\hat{\mathcal{F}}_{n}=\sigma\left(\hat{A}_{n}^{1}, \ldots, \hat{A}_{n}^{2^{n}}\right)$, $n=0, \ldots, N$, is generated by the time $n$ atoms

$$
\hat{A}_{n}^{j}=\left\{\omega_{k} \mid k \in\left\{1+(j-1) \frac{2^{N}}{2^{n}}, 2+(j-1) \frac{2^{N}}{2^{n}}, \ldots, j \frac{2^{N}}{2^{n}}\right\}\right\}, \quad j=1, \ldots, 2^{n} .
$$

The random variable $\hat{X}=\left(x_{1}, \ldots, x_{2^{N}}\right)$ on $(\hat{\Omega}, \hat{\mathcal{F}}, \hat{\mathbb{P}})$ has the same distribution as $X$ on $(\Omega, \mathcal{F}, \mathbb{P})$. Fix $k \in \mathbb{N}$ and define the stochastic process $\hat{Y}^{\varepsilon_{k}}$ inductively by $\hat{Y}_{N}^{\varepsilon_{k}}=\hat{X}$ and

$$
\hat{Y}_{n}^{\varepsilon_{k}}=u_{\gamma_{k}}^{-1} \circ \hat{\mathbb{E}}\left[u_{\gamma_{k}}\left(\hat{Y}_{n+1}^{\varepsilon_{k}}+2 \varepsilon_{k}\right) \mid \hat{\mathcal{F}}_{n}\right], \quad n=0, \ldots, N-1 .
$$

Let $\hat{y}_{n}^{1}<\cdots<\hat{y}_{n}^{2^{n}}$ denote the $2^{n}$ different values of the random variable $\hat{Y}_{n}^{\varepsilon_{k}}$, so that, by construction $\hat{A}_{n}^{j}=\left\{\hat{Y}_{n}^{\varepsilon_{k}}=\hat{y}_{n}^{j}\right\}$. Further, let $R^{\varepsilon_{k},+}$ be the stochastic process which follows the dynamics (19) and starts at $R_{0}^{\varepsilon_{k},+}=\hat{Y}_{0}^{\varepsilon_{k}}$.

Step 1. There exists an increasing sequence of a.s. finite stopping times $0=\tilde{\sigma}_{0} \leq \tilde{\sigma}_{1} \leq$ $\cdots \leq \tilde{\sigma}_{N}$ and for each $n=0, \ldots, N$ there is an almost sure partition $\left(A_{n}^{j}\right)_{j=1}^{2^{n}}$ of $\Omega$ such that

$$
\begin{gathered}
R_{\tilde{\sigma}_{n}}^{\varepsilon_{k},+} \geq \hat{y}_{n}^{j} \quad \text { on } A_{n}^{j}, \quad n=0, \ldots, N, \quad j=1, \ldots, 2^{n}, \\
\sum_{i=j}^{2^{n}} \mathbb{P}\left[A_{n}^{i}\right]>\sum_{i=j}^{2^{n}} \hat{\mathbb{P}}\left[\hat{A}_{n}^{i}\right], \quad n=1, \ldots, N, \quad j=2, \ldots, 2^{n} .
\end{gathered}
$$


In particular, $\mathbb{P}\left[R_{\tilde{\sigma}_{n}}^{\varepsilon_{k},+} \geq \hat{y}_{n}^{j}\right]>\hat{\mathbb{P}}\left[\hat{Y}_{n}^{\varepsilon_{k}} \geq \hat{y}_{n}^{j}\right]$ for all $n=1, \ldots, N, j=2, \ldots, 2^{n}$ and therefore

$$
R_{\tilde{\sigma}_{n}}^{\varepsilon_{k},+} \succeq_{1} \hat{Y}_{n}^{\varepsilon_{k}}, \quad n=0, \ldots, N .
$$

The proof of (30) and (31) is by induction on $n=0, \ldots, N$. By construction $R_{\tilde{\sigma}_{0}}^{\varepsilon_{k},+} \geq \hat{Y}_{0}^{\varepsilon_{k}}$. Fix $1 \leq m \leq N-1$. We assume that (30), (31) hold for all $n \leq m$ and we will show that there exists a finite stopping time $\tilde{\sigma}_{m+1} \geq \tilde{\sigma}_{m}$ and an almost sure partition $\left(A_{m+1}^{j}\right)_{j=1}^{2^{m+1}}$ of $\Omega$ such that (30), (31) also hold for $m+1$. Fix $1 \leq j_{0} \leq 2^{m}$. On $\hat{A}_{m}^{j_{0}}$, the random variable $\hat{Y}_{m}^{\varepsilon_{k}}$ equals $\hat{y}_{m}:=\hat{y}_{m}^{j_{0}}$, whereas $\hat{Y}_{m+1}^{\varepsilon_{k}}$ takes the two values $\hat{y}_{m+1}^{d}:=\hat{y}_{m+1}^{2 j_{0}-1}$ and $\hat{y}_{m+1}^{u}:=\hat{y}_{m+1}^{2 j_{0}}$. Then

$$
u_{\gamma_{k}}\left(\hat{y}_{m}\right)=\hat{p}^{d} u_{\gamma_{k}}\left(\hat{y}_{m+1}^{d}+2 \varepsilon_{k}\right)+\hat{p}^{u} u_{\gamma_{k}}\left(\hat{y}_{m+1}^{u}+2 \varepsilon_{k}\right),
$$

for the conditional probabilities $0<\hat{p}^{d}, \hat{p}^{u}<1$. On $A_{m}^{j_{0}}$, the stopping time $\tilde{\sigma}_{m+1}$ is defined by

$$
\tilde{\sigma}_{m+1}=\inf \left\{t \geq \tilde{\sigma}_{m} \mid R_{t}^{\varepsilon_{k},+} \in\left[\hat{y}_{m+1}^{d}, \hat{y}_{m+1}^{d}+2 \varepsilon_{k}\right) \text { or } R_{t}^{\varepsilon_{k},+} \geq \hat{y}_{m+1}^{u}\right\} .
$$

In view of (33) we deduce $\hat{y}_{m+1}^{d}+2 \varepsilon_{k}<\hat{y}_{m}<\hat{y}_{m+1}^{u}+2 \varepsilon_{k}$, which together with (30) yields $\hat{y}_{m+1}^{d}+\varepsilon_{k}<R_{\tilde{\sigma}_{m}}^{\varepsilon_{k},+}$. Due to Remark $3.4 u_{\varepsilon_{k}}\left(R^{\varepsilon_{k},+}\right)$ is a martingale. Hence the martingale convergence theorem implies that $\tilde{\sigma}_{m+1}$ is a.s. finite. Thus, the sets

$A_{m+1}^{2 j-1}=\left\{R_{\tilde{\sigma}_{m+1}}^{\varepsilon_{k},+} \in\left[\hat{y}_{m+1}^{2 j-1}, \hat{y}_{m+1}^{2 j-1}+2 \varepsilon_{k}\right)\right\} \quad$ and $\quad A_{m+1}^{2 j}=\left\{R_{\tilde{\sigma}_{m+1}}^{\varepsilon_{k},+} \geq \hat{y}_{m+1}^{2 j}\right\}, \quad j=1, \ldots 2^{m}$, form an almost sure partition of $\Omega$ and (30) holds for $n=m+1$. Since $u_{\gamma_{k}}\left(R^{\varepsilon_{k},+}\right)$ is a martingale, (30) yields $\mathbb{E}\left[u_{\gamma_{k}}\left(R_{\tilde{\sigma}_{m+1}}^{\varepsilon_{k},+}\right) \mid A_{m}^{j_{0}}\right] \geq u_{\varepsilon_{k}}\left(\hat{y}_{m}\right)$. If $R_{\tilde{\sigma}_{m}}^{\varepsilon_{k},+}>\hat{y}_{m+1}^{u}$, then we have $\mathbb{P}\left[R_{\tilde{\sigma}_{m+1}}^{\varepsilon_{k},+} \geq \hat{y}_{m+1}^{u} \mid A_{m}^{j_{0}}\right]=1$. If $R_{\tilde{\sigma}_{m}}^{\varepsilon_{k},+} \leq \hat{y}_{m+1}^{u}$, then $R_{\tilde{\sigma}_{m+1}}^{\varepsilon_{k},+} \leq \hat{y}_{m+1}^{u}+2 \varepsilon_{k}$, which in view of (33) and (34) leads to $\mathbb{P}\left[R_{\tilde{\sigma}_{m+1}}^{\varepsilon_{k},+} \geq \hat{y}_{m+1}^{u} \mid A_{m}^{j_{0}}\right]>\hat{p}^{u}$. This shows

$$
\mathbb{P}\left[R_{\tilde{\sigma}_{m+1}}^{\varepsilon_{k},+} \geq \hat{y}_{m+1}^{u} \mid A_{m}^{j_{0}}\right]>\hat{p}^{u} \quad \text { and } \quad \mathbb{P}\left[R_{\tilde{\sigma}_{m+1}}^{\varepsilon_{k},+} \geq \hat{y}_{m+1}^{d} \mid A_{m}^{j_{0}}\right] \geq 1 .
$$

We next prove (31) for $n=m+1$. By construction,

$$
\hat{\mathbb{P}}\left[\hat{A}_{m}^{j_{0}}\right] \hat{p}^{u}=\hat{\mathbb{P}}\left[\hat{A}_{m+1}^{2 j_{0}}\right] \quad \text { and } \quad \hat{\mathbb{P}}\left[\hat{A}_{m}^{j_{0}}\right]\left(1-\hat{p}^{u}\right)=\hat{\mathbb{P}}\left[\hat{A}_{m+1}^{2 j_{0}-1}\right] .
$$

On the one hand, if $\mathbb{P}\left[A_{m}^{j_{0}}\right] \geq \hat{\mathbb{P}}\left[\hat{A}_{m}^{j_{0}}\right]$, the induction hypothesis (31) and (35) imply

$$
\begin{aligned}
\sum_{i=2 j_{0}}^{2^{m+1}} \mathbb{P}\left[A_{m+1}^{i}\right] & =\mathbb{P}\left[A_{m}^{j_{0}}\right] \mathbb{P}\left[R_{\tilde{\sigma}_{m+1}}^{\varepsilon_{k},+} \geq \hat{y}_{m+1}^{2 j_{0}} \mid A_{m}^{j_{0}}\right]+\sum_{i=j_{0}+1}^{2^{m}} \mathbb{P}\left[A_{m}^{i}\right] \\
& >\hat{\mathbb{P}}\left[\hat{A}_{m}^{j_{0}}\right] \hat{p}^{u}+\sum_{i=j_{0}+1}^{2^{m}} \hat{\mathbb{P}}\left[\hat{A}_{m}^{i}\right]=\sum_{i=2 j_{0}}^{2^{m+1}} \hat{\mathbb{P}}\left[\hat{A}_{m}^{i}\right] .
\end{aligned}
$$


On the other hand, if $\mathbb{P}\left[A_{m}^{j_{0}}\right]<\hat{\mathbb{P}}\left[\hat{A}_{m}^{j_{0}}\right]$, we deduce

$$
\begin{aligned}
\sum_{i=1}^{2 j_{0}-1} \mathbb{P}\left[A_{m+1}^{i}\right] & =\mathbb{P}\left[A_{m}^{j_{0}}\right] \mathbb{P}\left[R_{\tilde{\sigma}_{m+1}}^{\varepsilon_{k},+}<\hat{y}_{m+1}^{2 j_{0}} \mid A_{m}^{j_{0}}\right]+\sum_{i=1}^{j_{0}-1} \mathbb{P}\left[A_{m}^{i}\right] \\
& <\hat{\mathbb{P}}\left[\hat{A}_{m}^{j_{0}}\right]\left(1-\hat{p}^{u}\right)+\sum_{i=1}^{j_{0}-1} \hat{\mathbb{P}}\left[\hat{A}_{m}^{i}\right]=\sum_{i=1}^{2 j_{0}-1} \hat{\mathbb{P}}\left[\hat{A}_{m}^{i}\right]
\end{aligned}
$$

This shows $\sum_{i=2 j_{0}}^{2^{m+1}} \mathbb{P}\left[A_{m+1}^{i}\right]>\sum_{i=2 j_{0}}^{2^{m+1}} \hat{\mathbb{P}}\left[\hat{A}_{m}^{i}\right]$. Moreover, if $j_{0} \geq 2$ (only in this case we have to check the induction hypothesis (31)), we deduce

$$
\sum_{i=2 j_{0}-1}^{2^{m+1}} \mathbb{P}\left[A_{m+1}^{i}\right]=\sum_{i=j_{0}}^{2^{m}} \mathbb{P}\left[A_{m}^{i}\right]>\sum_{i=j_{0}}^{2^{m}} \hat{\mathbb{P}}\left[\hat{A}_{m}^{i}\right]=\sum_{i=2 j_{0}-1}^{2^{m+1}} \hat{\mathbb{P}}\left[\hat{A}_{m}^{i}\right] .
$$

This completes the induction step.

Step 2. There is an increasing sequence of bounded stopping times $0=\sigma_{0} \leq \cdots \leq \sigma_{N}$ such that

$$
R_{\sigma_{n}}^{\varepsilon_{k},+} \succeq_{1} \hat{Y}_{n}^{\varepsilon_{k}}, \quad n=0, \ldots, N .
$$

Indeed, since (c.f. (31)) $\mathbb{P}\left[R_{\tilde{\sigma}_{n}}^{\varepsilon_{k},+} \geq \hat{y}_{n}^{j}\right]>\hat{\mathbb{P}}\left[\hat{Y}_{n}^{\varepsilon_{k}} \geq \hat{y}_{n}^{j}\right]$ for all $n=1, \ldots, N$ and all $j=$ $2, \ldots, 2^{n}$, the dominated convergence theorem implies $T \in \mathbb{N}$, such that

$$
\mathbb{P}\left[R_{\tilde{\sigma}_{n} \wedge T}^{\varepsilon_{k},+} \geq \hat{y}_{n}^{j}\right] \geq \hat{\mathbb{P}}\left[\hat{Y}_{n}^{\varepsilon_{k}} \geq \hat{y}_{n}^{j}\right] \quad \text { for all } n=1, \ldots, N \text { and all } j=2, \ldots, 2^{n} .
$$

Hence, for the bounded stopping times $\sigma_{n}=\tilde{\sigma}_{n} \wedge T$ we get (36).

Step 3. There is a subsequence of $\left(\varepsilon_{k}\right)$, which we still denote by $\left(\varepsilon_{k}\right)$, such that $\hat{Y}_{n}^{\varepsilon_{k}}$ converges to $\hat{Y}_{n}$ for all $n=0, \ldots, N-1$. Then, by continuity of $u_{\gamma}$ it follows $u_{\gamma_{k}}\left(\hat{Y}_{N-1}^{\varepsilon_{k}}\right) \rightarrow$ $u_{\gamma}\left(\hat{Y}_{N-1}\right)$ and $(29)$ yields

$$
u_{\gamma_{k}}\left(\hat{Y}_{N-1}^{\varepsilon_{k}}\right)=\hat{\mathbb{E}}\left[u_{\gamma_{k}}\left(\hat{X}+2 \varepsilon_{k}\right) \mid \hat{\mathcal{F}}_{N-1}\right] \rightarrow \hat{\mathbb{E}}\left[u_{\gamma}(\hat{X}) \mid \hat{\mathcal{F}}_{N-1}\right],
$$

showing that $u_{\gamma}\left(\hat{Y}_{N-1}\right)=\hat{\mathbb{E}}\left[u_{\gamma}(\hat{X}) \mid \hat{\mathcal{F}}_{N-1}\right]$. Backward induction yields

$u_{\gamma}\left(\hat{Y}_{n}\right)=\hat{\mathbb{E}}\left[u_{\gamma}(\hat{X}) \mid \hat{\mathcal{F}}_{n}\right] \quad$ and $\quad\left|u_{\gamma_{k}}\left(\hat{Y}_{n}^{\varepsilon_{k}}\right)-\hat{\mathbb{E}}\left[u_{\gamma_{k}}(\hat{X}) \mid \hat{\mathcal{F}}_{n}\right]\right| \rightarrow 0 \quad$ for all $n=0, \ldots, N-1$.

In particular, since $\hat{\mathbb{E}}\left[u_{\gamma_{k}}(\hat{X})\right]=\mathbb{E}\left[u_{\gamma_{k}}(X)\right]$, it follows

$$
\left|u_{\gamma_{k}}\left(R_{0}^{\varepsilon_{k},+}\right)-\mathbb{E}\left[u_{\gamma_{k}}(X)\right]\right| \rightarrow 0 .
$$

Using similar argument as before, there exist a stochastic processes $R^{\varepsilon_{k},-}$ of the form (19) and bounded stopping times $\sigma_{k}^{-}$such that $X \succeq R_{\sigma_{k}^{-}}^{\varepsilon_{k},-}$ and $\left|u_{\gamma_{k}}\left(R_{0}^{\varepsilon_{k},-}\right)-\mathbb{E}\left[u_{\gamma_{k}}(X)\right]\right| \rightarrow 0 . \square$

We now finish the proof of Theorem 1.4. We distinguish between three different cases. 
Case 1. There is a sequence $\left(\varepsilon_{k}\right)_{k \in \mathbb{N}}$ tending to zero such that $\gamma_{k}:=\gamma_{\varepsilon_{k}} \in \mathbb{R}$ and $\left(\gamma_{k}\right)_{k \in \mathbb{N}}$ converges to some $\gamma \in \mathbb{R}$ as $k$ tends to infinity. The sequence $\left(u_{\gamma_{k}}\right)_{k \in \mathbb{N}}$ then converges uniformly on compacts to the function $u_{\gamma}$. Let $X$ be a random variable in $L^{\infty}$. $\rho_{0}$ is monotone with respect to $\succeq_{1}$. Indeed, let $U$ be a random variable that is uniformly distributed on $(0,1)$. If $Y_{1}, Y_{2}$ are random variables such that $Y_{1} \succeq_{1} Y_{2}$, then $F_{Y_{1}}^{-1}(U)$ has the same distribution as $Y_{1}, F_{Y_{2}}^{-1}(U)$ has the same distribution as $Y_{2}$, and $F_{Y_{1}}^{-1}(U) \geq F_{Y_{2}}^{-1}(U)$. Since $\rho_{0}$ is law invariant, one obtains that $\geq$-monotonicity implies $\succeq_{1}$-monotonicity. Hence, Lemma 2.3 and Lemma 2.5 imply

$$
\rho_{0}(X) \geq \rho_{0}\left(R_{\sigma_{k}^{+}}^{\varepsilon_{k},+}\right)=-R_{0}^{\varepsilon_{k},+}=-\lim _{k \rightarrow \infty} u_{\gamma_{k}}^{-1} \circ \mathbb{E}\left[u_{\gamma_{k}}(X)\right]=-u_{\gamma}^{-1} \circ \mathbb{E}\left[u_{\gamma}(X)\right] .
$$

Analogously, it follows $\rho_{0}(X) \leq-u_{\gamma}^{-1} \circ \mathbb{E}\left[u_{\gamma}(X)\right]$, showing that $\rho_{0}(X)=\rho^{\gamma}(X)$.

Case 2. There is a sequence $\left(\varepsilon_{k}\right)_{k \in \mathbb{N}}$ tending to zero such that $\liminf _{k \rightarrow \infty} \gamma_{k}=+\infty$. Let $X \in L^{\infty}$, fix $M \in \mathbb{N}$ and define $\tilde{\gamma}_{k}:=\gamma_{k} \wedge M$ with respective $\tilde{\eta}_{\varepsilon_{k}}:=\rho^{\tilde{\gamma}_{k}}\left(\varepsilon_{k} b_{1}\right)$. By Lemma 2.5 there exist $\tilde{R}^{\varepsilon_{k}}$ of the form (19) and a bounded stopping times $\tilde{\sigma}_{k}$ such that

$$
\tilde{R}_{\tilde{\sigma}_{k}}^{\varepsilon_{k}} \succeq_{1} X \quad \text { and } \quad \lim _{k \rightarrow \infty}\left|u_{\tilde{\gamma}_{k}}\left(\tilde{R}_{0}^{\varepsilon_{k}}\right)-\mathbb{E}\left[u_{\tilde{\gamma}_{k}}(X)\right]\right|=0 .
$$

Define $R_{t}^{\varepsilon_{k}}:=\tilde{R}_{0}^{\varepsilon_{k}}+\sum_{j=1}^{t}\left(\varepsilon_{k} b_{j}+\eta_{\varepsilon_{k}}\right)$. Since $\tilde{\gamma}_{k} \leq \gamma_{k}$, Lemma 2.1 (iv) implies $\tilde{\eta}_{\varepsilon_{k}} \leq \eta_{\varepsilon_{k}}$ and whence $R_{\tilde{\sigma}_{k}}^{\varepsilon_{k}} \succeq_{1} \tilde{R}_{\tilde{\sigma}_{k}}^{\varepsilon_{k}}$. Lemma 2.3 in combination with (38) yields

$$
\rho_{0}(X) \geq \limsup _{k \rightarrow \infty} \rho_{0}\left(R_{\tilde{\sigma}_{k}}^{\varepsilon_{k}}\right)=-\liminf _{k \rightarrow \infty} R_{0}^{\varepsilon_{k}}=-\liminf _{k \rightarrow \infty} \tilde{R}_{0}^{\varepsilon_{k}}=-u_{M}^{-1} \circ \mathbb{E}\left[u_{M}(X)\right]=\rho^{M}(X) .
$$

Taking the limes $M \rightarrow \infty$ we deduce $\rho_{0}(X) \geq \sup _{Z \in \mathcal{P}_{0}} \mathbb{E}[Z(-X)]$ from Lemma 2.1 (i). Hence, $\rho_{0}$ is dominated below by the worst case risk measure, that is, $\rho_{0}$ has to be the worst case risk measure itself.

Case 3. If there is a sequence $\left(\varepsilon_{k}\right)_{k \in \mathbb{N}}$ tending to zero such that $\lim _{\sup _{k \rightarrow \infty}} \gamma_{k}=-\infty$, then similar arguments as given in case 2 imply that $\rho_{0}(X)=\inf _{Z \in \mathcal{P}_{0}} \mathbb{E}[Z(-X)]$. However, the best case risk measure $\inf _{Z \in \mathcal{P}_{0}} \mathbb{E}[Z(-X)]$ is not relevant. Hence, the case 3 is excluded and we are left with either case 1 or case 2 . The proof is completed.

\section{Proof of Theorem 1.4}

Throughout this section, $\left(\Omega, \mathcal{F},\left(\mathcal{F}_{t}\right)_{t \in \mathbb{N}_{0}}, \mathbb{P}\right)$ is a standard filtered probability space.

Proof of the "if"-part of Theorem 1.4.

Let us assume that $c_{0}: L^{\infty}(a, b) \rightarrow \mathbb{R}$ is of the form $u^{-1} \circ \mathbb{E}[u(X)]$, where $u:(a, b) \rightarrow \mathbb{R}$ is a strictly increasing, continuous function. Obviously, $c_{0}$ is normalized on constants, strictly monotone, $\|\cdot\|_{\infty}$-continuous, law invariant and satisfies condition (C). Moreover, for $t \in \mathbb{N}$ we define $c_{t}(X):=u^{-1} \circ \mathbb{E}\left[u(X) \mid \mathcal{F}_{t}\right]$ and deduce on $A \in \mathcal{F}_{t}$

$$
1_{A} c_{t}(X)=1_{A} u^{-1} \circ \mathbb{E}\left[u(X) \mid \mathcal{F}_{t}\right]=1_{A} u^{-1} \circ \mathbb{E}\left[u(Y) \mid \mathcal{F}_{t}\right]=1_{A} c_{t}(Y),
$$


for all $X, Y \in L^{\infty}(a, b)$ with $X 1_{A}=Y 1_{A}$. Moreover,

$$
c_{0}\left(c_{t}(X)\right)=u^{-1} \circ \mathbb{E}\left[u\left(u^{-1} \circ \mathbb{E}\left[u(X) \mid \mathcal{F}_{t}\right]\right)\right]=c_{0}(X),
$$

showing that $c_{0}$ is time consistent.

Preparations for the "only if"-part of Theorem 1.4.

Suppose that $c_{0}: L^{\infty}(a, b) \rightarrow \mathbb{R}$ is normalized on constants, strictly monotone, $\|\cdot\|_{\infty^{-}}$ continuous, law invariant, time consistent and satisfies condition (C). Lemma 2.2 specializes in the present context as follows.

Lemma 3.1 Let $t \in \mathbb{N}$ and $X, Y \in L^{\infty}(a, b)$ with $\operatorname{law}(X)=\operatorname{law}(Y)$ a.s. and $Y$ is independent of $\mathcal{F}_{t}$. Then, we have $c_{0}(X)=c_{t}(Y)$.

Proof. If $c_{t}(Y)$ is constant, then, since $c_{0}$ is normalized on constants, time consistent, law invariant and $\operatorname{law}(X)=\operatorname{law}(Y)$ a.s., it follows

$$
c_{t}(Y)=c_{0}\left(c_{t}(Y)\right)=c_{0}(Y)=c_{0}(X) .
$$

We therefore assume that $c_{t}(Y)$ is not constant. If $c_{t}(Y) \leq c_{0}(X)$ and $\mathbb{P}\left[c_{t}(Y)<c_{0}(X)\right]>0$ then strict monotonicity and time consistency of $c_{0}$ yield $c_{0}(Y)=c_{0}\left(c_{t}(Y)\right)<c_{0}(X)$ which is a contradiction. Analogously, $c_{t}(Y) \geq c_{0}(X)$ and $\mathbb{P}\left[c_{t}(Y)>c_{0}(X)\right]>0$ is absurd. Thus, there exist $A, A^{\prime} \in \mathcal{F}_{t}$ with $\mathbb{P}[A]=\mathbb{P}\left[A^{\prime}\right]>0$ such that $c_{t}(Y)<c_{0}(X)$ on $A$ and $c_{t}(Y)>$ $c_{0}(X)$ on $A^{\prime}$. In view of the local property of $c_{t}$, time consistency and strict monotonicity of $c_{0}$ we deduce for $m \in(a, b)$

$$
\begin{gathered}
c_{0}\left(1_{A} Y+1_{A^{c}} m\right)=c_{0}\left(c_{t}\left(1_{A} Y+1_{A^{c}} m\right)\right)=c_{0}\left(1_{A} c_{t}(Y)+1_{A^{c}} m\right)<c_{0}\left(1_{A} c_{0}(X)+1_{A^{c}} m\right) \\
c_{0}\left(1_{A^{\prime}} Y+1_{A^{\prime c}} m\right)=c_{0}\left(c_{t}\left(1_{A^{\prime}} Y+1_{A^{\prime c}} m\right)\right)=c_{0}\left(1_{A^{\prime}} c_{t}(Y)+1_{A^{\prime c}} m\right)>c_{0}\left(1_{A^{\prime}} c_{0}(X)+1_{A^{\prime c}} m\right) .
\end{gathered}
$$

On the other hand, law $\left(1_{A} Y+1_{A^{c}} m\right)=\operatorname{law}\left(1_{A^{\prime}} Y+1_{A^{\prime c}} m\right)$ a.s. as well as law $\left(1_{A} c_{0}(X)+\right.$ $\left.1_{A^{c}} m\right)=\operatorname{law}\left(1_{A^{\prime}} c_{0}(X)+1_{A^{\prime c}} m\right)$ a.s., which in view of (39) and (40) is a contradiction to the law invariance of $c_{0}$. This shows that $c_{t}(Y)$ is a constant and whence $c_{0}(X)=c_{t}(Y)$.

Let us fix a compact interval $[A, B] \subset(a, b)$ for some $A, B \in \mathbb{R}$ with $A<B$. For any $\varepsilon_{n}:=(B-A) / n, n \in \mathbb{N}$, we define

$$
\mathcal{I}_{\varepsilon_{n}}=\left\{A+\varepsilon_{n}, A+2 \varepsilon_{n}, \ldots, A+(n-2) \varepsilon_{n}, A+(n-1) \varepsilon_{n}\right\} .
$$

Lemma 3.2 For all $\varepsilon_{n}$ and all $x \in\left[A+\varepsilon_{n}, B-\varepsilon_{n}\right]$ there exist Bernoulli random variables $b_{+}^{\varepsilon_{n}}(x)$ and $b_{-}^{\varepsilon_{n}}(x)$ taking the values $+\varepsilon_{n}$ and $-\varepsilon_{n}$ with probabilities

$$
\begin{array}{lll}
\mathbb{P}\left[b_{+}^{\varepsilon_{n}}(x)=\varepsilon_{n}\right]=p_{+}^{\varepsilon_{n}}(x) \quad \text { and } & \mathbb{P}\left[b_{+}^{\varepsilon_{n}}(x)=-\varepsilon_{n}\right]=1-p_{+}^{\varepsilon_{n}}(x), \\
\mathbb{P}\left[b_{-}^{\varepsilon_{n}}(x)=\varepsilon_{n}\right]=p_{-}^{\varepsilon_{n}}(x) \quad \text { and } \quad \mathbb{P}\left[b_{-}^{\varepsilon_{n}}(x)=-\varepsilon_{n}\right]=1-p_{-}^{\varepsilon_{n}}(x),
\end{array}
$$


as well as increasing, continuous functions $u_{\varepsilon_{n}}^{+}:[A, B] \rightarrow[0,1]$ and $u_{\varepsilon_{n}}^{-}:[A, B] \rightarrow[0,1]$ such that

$$
\begin{gathered}
c_{0}\left(x+b_{+}^{\varepsilon_{n}}(x)\right) \leq x, \quad c_{0}\left(x+b_{-}^{\varepsilon_{n}}(x)\right) \geq x, \quad \text { for all } x \in\left[A+\varepsilon_{n}, B-\varepsilon_{n}\right], \\
u_{\varepsilon_{n}}^{+}(x)=\mathbb{E}\left[u_{\varepsilon_{n}}^{+}\left(x+b_{+}^{\varepsilon_{n}}(x)\right)\right], \quad u_{\varepsilon_{n}}^{-}(x)=\mathbb{E}\left[u_{\varepsilon_{n}}^{-}\left(x+b_{-}^{\varepsilon_{n}}(x)\right)\right], \quad \text { for all } x \in \mathcal{I}_{\varepsilon_{n}},
\end{gathered}
$$

and

$$
\left\|u_{\varepsilon_{n}}^{+}-u_{\varepsilon_{n}}^{-}\right\|_{\infty} \rightarrow 0
$$

Proof. Fix $\varepsilon_{n}$. For any $x \in\left[A+\varepsilon_{n}, B-\varepsilon_{n}\right]$ we define

$$
p(x):=\sup \left\{p \in[0,1] \mid c_{0}\left(x+b^{\varepsilon, p}\right) \leq x\right\} .
$$

Recall that $b^{\varepsilon, p}$ denotes a Bernoulli random variable taking the values $\varepsilon$ and $-\varepsilon$ with probabilities $p$ and $1-p$. Condition $(\mathrm{C})$ implies that $p(x) \in(0,1]$.

Step 1. There is an increasing, continuous function $u_{\varepsilon_{n}}:[A, B] \rightarrow[0,1]$ such that $u_{\varepsilon_{n}}(A)=0, u_{\varepsilon_{n}}(B)=1$ and

$$
u_{\varepsilon_{n}}(x)=\mathbb{E}\left[u_{\varepsilon_{n}}\left(x+b^{\varepsilon_{n}, p(x)}\right)\right], \quad \text { for all } x \in \mathcal{I}_{\varepsilon_{n}} .
$$

Indeed, define $\tilde{u}_{\varepsilon_{n}}(A)=0, \tilde{u}_{\varepsilon_{n}}\left(A+\varepsilon_{n}\right)=1$ and inductively

$$
\tilde{u}_{\varepsilon_{n}}\left(A+(k+1) \varepsilon_{n}\right):=\frac{1}{p\left(k \varepsilon_{n}\right)}\left[\tilde{u}_{\varepsilon_{n}}\left(A+k \varepsilon_{n}\right)-\left(1-p\left(k \varepsilon_{n}\right)\right) \tilde{u}_{\varepsilon_{n}}\left(A+(k-1) \varepsilon_{n}\right)\right]
$$

for all $k=1, \ldots, n-1$. Then $\tilde{u}_{\varepsilon_{n}}(x) \geq \tilde{u}_{\varepsilon_{n}}(y)$ for all $x, y \in \mathcal{I}_{\varepsilon_{k}}$ with $x \geq y$. The normalized function $u_{\varepsilon_{n}}(x):=\tilde{u}_{\varepsilon_{n}}(x) / \tilde{u}_{\varepsilon_{n}}(B), x \in \mathcal{I}_{\varepsilon_{n}} \cup\{A, B\}$, then satisfies $u_{\varepsilon_{n}}(A)=0, u_{\varepsilon_{n}}(B)=1$ and (45). By linear interpolation $u_{\varepsilon_{n}}$ extends to an increasing function on $[A, B]$, i.e., for $A+k \varepsilon_{n}<x<A+(k+1) \varepsilon_{n}$ we define

$$
u_{\varepsilon_{n}}(x):=u_{\varepsilon_{n}}\left(A+k \varepsilon_{n}\right)+\frac{u_{\varepsilon_{n}}\left(A+(k+1) \varepsilon_{n}\right)-u_{\varepsilon_{n}}\left(A+k \varepsilon_{n}\right)}{\varepsilon_{n}}\left(x-\left(A+k \varepsilon_{n}\right)\right) .
$$

Step 2. Let $\left(p_{k}^{+}\right)_{k \in \mathbb{N}}$ be a sequence satisfying $0<p_{k}^{+}(x)<p(x)$ and $p_{k}^{+}(x) \nearrow p(x)$ for all $x \in\left[A+\varepsilon_{n}, B-\varepsilon_{n}\right]$. Due to (44) we have $c_{0}\left(x+b^{\varepsilon_{n}, p_{k}^{+}(x)}\right) \leq x$ for all $x \in\left[A+\varepsilon_{n}, B-\varepsilon_{n}\right]$. Let $u_{\varepsilon_{k}}^{k,+}$ denote the increasing, continuous function constructed in Step 1 associated to $p_{k}^{+}(x)$. Then

$$
u_{\varepsilon_{n}}^{k,+}(x)=\mathbb{E}\left[u_{\varepsilon_{n}}^{k,+}\left(x+b^{\varepsilon_{n}, p_{k}^{+}(x)}\right)\right], \quad \text { for all } x \in \mathcal{I}_{\varepsilon_{n}} .
$$

Further, since $p_{k}^{+}(x) \nearrow p(x)$ for all $x \in \mathcal{I}_{\varepsilon_{n}}$, the sequence $\left(u_{\varepsilon_{n}}^{k,+}\right)_{k \in \mathbb{N}}$ converges uniformly on $[A, B]$ to $u_{\varepsilon_{n}}$. Hence, there is $k_{0} \in \mathbb{N}$ such that

$$
b_{+}^{\varepsilon_{n}}(x):=b^{\varepsilon_{n}, p_{k_{0}}^{+}(x)} \quad \text { and } \quad u_{\varepsilon_{n}}^{+}(x):=u_{\varepsilon_{k}}^{k_{0},+}(x)
$$

satisfy (41), (42) and $\left\|u_{\varepsilon_{n}}^{+}-u_{\varepsilon_{n}}\right\|_{\infty} \leq \varepsilon_{n}$. Analogously, there exists a sequence $p(x) \leq$ $p_{k}^{-}(x) \leq 1$ with $p_{k}^{-}(x) \rightarrow p(x)$ for all $x \in\left[A+\varepsilon_{n}, B-\varepsilon_{n}\right]$ and $c_{0}\left(x+b^{\varepsilon_{n}, p_{k}^{-}(x)}\right) \geq x$ for all 
$x \in\left[A+\varepsilon_{n}, B-\varepsilon_{n}\right]$. Note that strict monotonicity of $c_{0}$ yields $c_{0}\left(x+b^{\varepsilon_{n}, 1}\right)>x$. Let $u_{\varepsilon_{k}}^{k,-}$ denote the increasing, continuous function constructed in Step 1 associated to $p_{k}^{-}(x)$. The sequence $\left(u_{\varepsilon_{n}}^{k,-}\right)_{k \in \mathbb{N}}$ converges uniformly on $[A, B]$ to $u_{\varepsilon_{n}}$. Again, there is $k_{0} \in \mathbb{N}$ such that

$$
b_{-}^{\varepsilon_{n}}(x):=b^{\varepsilon_{n}, p_{k_{0}}^{-}(x)} \quad \text { and } \quad u_{\varepsilon_{n}}^{-}(x):=u_{\varepsilon_{k}}^{k_{0},-}(x)
$$

satisfy (41), (42) and $\left\|u_{\varepsilon_{n}}^{-}-u_{\varepsilon_{n}}\right\|_{\infty} \leq \varepsilon_{n}$. Together, $\left\|u_{\varepsilon_{n}}^{+}-u_{\varepsilon_{n}}^{-}\right\|_{\infty} \leq 2 \varepsilon_{n} \rightarrow 0$ as $n \rightarrow \infty$.

In view of Lemma A.1 there exists a subsequence of $\left(\varepsilon_{n}\right)$ which we denote by $\left(\varepsilon_{k}\right)$ such that $\left(u_{\varepsilon_{k}}^{+}\right)$and $\left(u_{\varepsilon_{k}}^{-}\right)$converge pointwise to an increasing function $u:[A, B] \rightarrow[0,1]$. For fixed $k$ and $t \in \mathbb{N}_{0}$ and any $\mathcal{F}_{t}$-measurable random variable $X$ with values in $\left\{x_{1}, \ldots, x_{N}\right\}$, $x_{i} \in\left[A+\varepsilon_{k}, B-\varepsilon_{k}\right]$, let $b_{t+1}^{\varepsilon_{k}, \pm}(X)$ denote the $\mathcal{F}_{t+1}$-measurable random variables $b_{t+1}^{\varepsilon_{k},+}(X)$ and $b_{t+1}^{\varepsilon_{k},-}(X)$ with distribution

$$
\operatorname{law}\left(b_{t+1}^{\varepsilon_{k}, \pm}(X) \mid X=x\right)=\operatorname{law}\left(b_{ \pm}^{\varepsilon_{k}}(x)\right) \text { for all } x \in\left\{x_{1}, \ldots, x_{N}\right\} .
$$

Let $\left(R_{t}^{\varepsilon_{k}, \pm}\right)_{t \in \mathbb{N}_{0}}$ denote the stopped random walks which start at $R_{0}^{\varepsilon_{k}, \pm} \in[A, B]$ and follows the dynamics

$$
R_{t+1}^{\varepsilon_{k}, \pm}= \begin{cases}R_{t}^{\varepsilon_{k}, \pm}+b_{t+1}^{\varepsilon_{k}, \pm}\left(R_{t}^{\varepsilon_{k}, \pm}\right) & \text { if } t<\tau_{0}^{ \pm} \\ R_{\tau_{0}}^{\varepsilon_{k}, \pm} & \text { if } t \geq \tau_{0}^{ \pm}\end{cases}
$$

for the stopping times $\tau_{0}^{ \pm}=\inf \left\{t \in \mathbb{N}_{0} \mid R_{t}^{\varepsilon_{k}, \pm} \in\left[A, A+\varepsilon_{k}\right]\right.$ or $\left.R_{t}^{\varepsilon_{k}, \pm} \in\left[B-\varepsilon_{k}, B\right]\right\}$, where the infimum over the empty set is defined as $+\infty$. By construction, $R^{\varepsilon_{k}, \pm}$ are Markov processes with values in $\left(R_{0}^{\varepsilon_{k}, \pm}+\varepsilon_{k} \mathbb{N}\right) \cap[A, B]$ and transition probabilities

$$
\begin{gathered}
\mathbb{P}\left[R_{t+1}^{\varepsilon_{k}, \pm}=x+\varepsilon_{k} \mid R_{t}^{\varepsilon_{k}, \pm}=x\right]=p_{ \pm}^{\varepsilon_{k}}(x), \\
\mathbb{P}\left[R_{t+1}^{\varepsilon_{k}, \pm}=x-\varepsilon_{k} \mid R_{t}^{\varepsilon_{k}, \pm}=x\right]=1-p_{ \pm}^{\varepsilon_{k}}(x) .
\end{gathered}
$$

Lemma 2.3 specializes to the present context as follows. The proof is a straightforward modification.

Lemma 3.3 Let $R^{\varepsilon_{k}, \pm}$ denote the stochastic processes which follow the dynamics (46) and let $\tau$ be a bounded stopping time. Then, we have

$$
c_{0}\left(R_{\tau}^{\varepsilon_{k},+}\right) \leq R_{0}^{\varepsilon_{k},+} \quad \text { and } \quad c_{0}\left(R_{\tau}^{\varepsilon_{k},-}\right) \geq R_{0}^{\varepsilon_{k},-} .
$$

Proof. Suppose that $R_{s}^{\varepsilon_{k},+}$ assumes the values $\left\{x_{1}, \ldots, x_{N}\right\}$. Due to (46) we have

$$
R_{s+1}^{\varepsilon_{k},+}=1_{\left\{s \geq \tau_{0}^{+}\right\}} R_{s}^{\varepsilon_{k},+}+1_{\left\{s+1 \leq \tau_{0}^{+}\right\}} \sum_{n=1}^{N} 1_{\left\{R_{s}^{\varepsilon_{k},+}=x_{n}\right\}}\left(x_{n}+b_{s+1}^{\varepsilon_{k},+}\left(x_{n}\right)\right) .
$$

The local property of $c_{s}$ yields

$$
c_{s}\left(R_{s+1}^{\varepsilon_{k},+}\right)=1_{\left\{s \geq \tau_{0}^{+}\right\}} c_{s}\left(R_{s}^{\varepsilon_{k},+}\right)+1_{\left\{s+1 \leq \tau_{0}^{+}\right\}} \sum_{n=1}^{N} 1_{\left\{R_{s}^{\varepsilon_{k},+}=x_{n}\right\}} c_{s}\left(x_{n}+b_{s+1}^{\varepsilon_{k},+}\left(x_{n}\right)\right) .
$$


Since law $\left(x_{n}+b_{s+1}^{\varepsilon_{k},+}\left(x_{n}\right) \mid \mathcal{F}_{s}\right)=\operatorname{law}\left(x_{n}+b_{+}^{\varepsilon_{k}}\left(x_{n}\right)\right)$ a.s., Lemma 3.1 and Lemma 3.2 yield

$$
c_{s}\left(x_{n}+b_{s+1}^{\varepsilon_{k},+}\left(x_{n}\right)\right)=c_{0}\left(x_{n}+b_{+}^{\varepsilon_{k}}\left(x_{n}\right)\right) \leq x_{n} .
$$

We get $c_{s}\left(R_{s+1}^{\varepsilon_{k},+}\right) \leq R_{s}^{\varepsilon_{k},+}$ from (47) and (48). We then proceed by backward induction as in the proof of Lemma 2.3.

Remark 3.4 For any $R^{\varepsilon_{k}, \pm}$ of the form (46) starting at $R_{0}^{\varepsilon_{k}, \pm} \in \mathcal{I}_{\varepsilon_{k}}$, the stochastic processes $u_{\varepsilon_{k}}^{ \pm}\left(R_{t}^{\varepsilon_{k}, \pm}\right)$ are martingales. Indeed, since $\mathbb{P}\left[R_{t}^{\varepsilon_{k}, \pm} \in \mathcal{I}_{\varepsilon_{k}}\right]=1$ for all $t \in \mathbb{N}_{0}$ we deduce from (42) that

$$
\begin{aligned}
\mathbb{E}\left[u_{\varepsilon_{k}}^{ \pm}\left(R_{t+1}^{\varepsilon_{k}, \pm}\right) \mid \mathcal{F}_{t}\right]= & 1_{\left\{t<\tau_{0}^{ \pm}\right\}} \mathbb{E}\left[u_{\varepsilon_{k}}^{ \pm}\left(R_{t}^{\varepsilon_{k}, \pm}+b_{t+1}^{\varepsilon_{k}, \pm}\left(R_{t}^{\varepsilon_{k}, \pm}\right)\right) \mid \mathcal{F}_{t}\right] \\
& +1_{\left\{t \geq \tau_{0}^{ \pm}\right\}} \mathbb{E}\left[u_{\varepsilon_{k}}^{ \pm}\left(R_{t}^{\varepsilon_{k}, \pm}\right) \mid \mathcal{F}_{t}\right]=u_{\varepsilon_{k}}^{ \pm}\left(R_{t}^{\varepsilon_{k}, \pm}\right) .
\end{aligned}
$$

Lemma 3.5 The function $u:[A, B] \rightarrow[0,1]$ is continuous and strictly increasing. For any random variable $X$ taking at most two values $x_{1}, x_{2} \in(A, B)$, we have

$$
c_{0}(X)=u^{-1} \circ \mathbb{E}[u(X)] .
$$

Proof. Step 1. Suppose that $X$ assumes the values $A<x_{1}<x_{2}<B$ with probabilities $0<p_{1}, p_{2}<1$. Let

$$
f_{\varepsilon_{k}}^{+}(x):=\left(u_{\varepsilon_{k}}^{+}\right)^{-1}\left\{p_{1} u_{\varepsilon_{k}}^{+}\left(x_{1}+x\right)+p_{2} u_{\varepsilon_{k}}^{+}\left(x_{2}+x\right)\right\}
$$

be a sequence of functions defined on $[-\kappa, \kappa]$ for some $\kappa>0$. The functions $f_{\varepsilon_{k}}^{+}$are continuous, increasing and bounded by $x_{1}-\kappa \leq f_{\varepsilon_{k}}^{+}(x) \leq x_{2}+\kappa$ for all $x \in[-\kappa, \kappa]$. In view of Lemma A.1, there exists a subsequence of $\left(\varepsilon_{k}\right)$, which we still denote by $\left(\varepsilon_{k}\right)$, such that $f_{\varepsilon_{k}}^{+}$converges pointwise to an increasing function $f^{+}:[-\kappa, \kappa] \rightarrow\left[x_{1}-\kappa, x_{2}+\kappa\right]$. In this first step, we assume in addition that 0 is a continuity point of $f^{+}$. Define

$$
Y_{0}^{\varepsilon_{k}}:=\left(u_{\varepsilon_{k}}^{+}\right)^{-1}\left\{p_{1} u_{\varepsilon_{k}}^{+}\left(x_{1}+\varepsilon_{k}\right)+p_{2} u_{\varepsilon_{k}}^{+}\left(x_{2}+\varepsilon_{k}\right)\right\} \in\left[x_{1}+\varepsilon_{k}, x_{2}+\varepsilon_{k}\right] .
$$

Here we assume that $k$ is large enough such that $x_{2}+2 \varepsilon_{k}<B$. Let $R^{\varepsilon_{k},+}$ be defined as the stochastic process which follows the dynamics (46) and starts at

$$
R_{0}^{\varepsilon_{k},+}:=\min \left\{x \in \mathcal{I}_{\varepsilon_{k}} \mid x \geq Y_{0}^{\varepsilon_{k}}\right\} \in\left[x_{1}+\varepsilon_{k}, x_{2}+2 \varepsilon_{k}\right] .
$$

Define the stopping time

$$
\sigma:=\inf \left\{t \geq 0 \mid R_{t}^{\varepsilon_{k},+} \in\left[x_{1}, x_{1}+\varepsilon_{k}\right) \text { or } R_{t}^{\varepsilon_{k},+} \geq x_{2}\right\} .
$$

Due to Remark 3.4, the process $u_{\varepsilon_{k}}^{+}\left(R_{t}^{\varepsilon_{k},+}\right)$ is a martingale. Hence, the martingale convergence theorem yields that $\sigma$ is a.s. finite. If $R_{0}^{\varepsilon_{k},+} \geq x_{2}$ then $\mathbb{P}\left[R_{\sigma}^{\varepsilon_{k},+} \geq x_{2}\right]=1$. If $R_{0}^{\varepsilon_{k},+}<x_{2}$ then $R_{\sigma}^{\varepsilon_{k},+}<x_{2}+\varepsilon_{k}$ and the martingale stopping theorem yields

$$
p_{1} u_{\varepsilon_{k}}^{+}\left(x_{1}+\varepsilon_{k}\right)+p_{2} u_{\varepsilon_{k}}^{+}\left(x_{2}+\varepsilon_{k}\right) \leq u_{\varepsilon_{k}}^{+}\left(R_{0}^{\varepsilon_{k},+}\right)=\mathbb{E}\left[u_{\varepsilon_{k}}^{+}\left(Y_{\sigma}^{\varepsilon_{k},+}\right)\right] .
$$


Hence, $\mathbb{P}\left[R_{\sigma}^{\varepsilon_{k},+} \geq x_{2}\right]>p_{2}$. The dominating convergence theorem implies $T \in \mathbb{N}$ such that $\mathbb{P}\left[R_{\sigma \wedge T}^{\varepsilon_{k},+} \geq x_{2}\right] \geq p_{2}$. Hence, for the bounded stopping time $\sigma_{+}^{k}:=\sigma \wedge T$ we end up with

$$
R_{\sigma_{+}^{k}}^{\varepsilon_{k},+} \succeq_{1} X
$$

Since 0 is a continuity point of $f^{+}$we deduce from Lemma A.1

$$
\left|\left(u_{\varepsilon_{k}}^{+}\right)^{-1}\left\{p_{1} u_{\varepsilon_{k}}^{+}\left(x_{1}+\varepsilon_{k}\right)+p_{2} u_{\varepsilon_{k}}^{+}\left(x_{2}+\varepsilon_{k}\right)\right\}-\left(u_{\varepsilon_{k}}^{+}\right)^{-1}\left\{p_{1} u_{\varepsilon_{k}}^{+}\left(x_{1}\right)+p_{2} u_{\varepsilon_{k}}^{+}\left(x_{2}\right)\right\}\right| \rightarrow 0
$$

as $k \rightarrow \infty$, and therefore in combination with (50) and $\left|R_{0}^{\varepsilon_{k},+}-Y_{0}^{\varepsilon_{k}}\right| \leq \varepsilon_{k}$, we deduce

$$
\left|R_{0}^{\varepsilon_{k},+}-\left(u_{\varepsilon_{k}}^{+}\right)^{-1} \circ \mathbb{E}\left[u_{\varepsilon_{k}}^{+}(X)\right]\right| \rightarrow 0 \quad \text { as } k \rightarrow \infty .
$$

Analogously, if 0 is a continuity point of $f^{-}$defined as a converging subsequence of

$$
f_{\varepsilon_{k}}^{-}(x):=\left(u_{\varepsilon_{k}}^{-}\right)^{-1}\left\{p_{1} u_{\varepsilon_{k}}^{-}\left(x_{1}+x\right)+p_{2} u_{\varepsilon_{k}}^{-}\left(x_{2}+x\right)\right\},
$$

then there exist $R^{\varepsilon_{k},-}$ of the form (46) starting at $R_{0}^{\varepsilon_{k},-} \in \mathcal{I}_{\varepsilon_{k}}$ and a bounded stopping times $\sigma_{k}^{-}$such that $X \succeq_{1} R_{\sigma_{k}^{-}}^{\varepsilon_{k},-}$ and $\left|R_{0}^{\varepsilon_{k},-}-\left(u_{\varepsilon_{k}}^{-}\right)^{-1} \circ \mathbb{E}\left[u_{\varepsilon_{k}}^{-}(X)\right]\right| \rightarrow 0$.

Step 2. Suppose that $X$ takes the values $A<x_{1}<x_{2}<B$ with probabilities $0<$ $p_{1}, p_{2}<1$, such that $c_{0}(X)$ is a continuity point of the function $u$. Fix $\delta>0$. There exists $x \in(-\kappa, 0)$ such that $X^{\delta}$ taking the values $x_{1}^{\delta}=x_{1}+x$ and $x_{2}^{\delta}=x_{2}+x$ with probabilities $p_{1}$ and $p_{2}$ satisfies

(i) $\left|c_{0}(X)-c_{0}\left(X^{\delta}\right)\right| \leq \delta$,

(ii) $c_{0}\left(X^{\delta}\right)$ is a continuity point of $u$,

(iii) $x$ is a continuity point of $f^{+}$,

(iv) $X \geq X^{\delta}$ and $\left\|X^{\delta}-X\right\|_{\infty} \leq \delta$.

Due to Step 1, there are $\left(R^{\varepsilon_{k},+}\right)$ and $\left(\sigma_{k}^{+}\right)$such that (51) and (52) hold, i.e.,

$$
R_{\sigma_{k}^{+}}^{\varepsilon_{k},+} \succeq_{1} X^{\delta} \quad \text { and } \quad \xi_{k}:=\left|R_{0}^{\varepsilon_{k},+}-\left(u_{\varepsilon_{k}}^{+}\right)^{-1} \circ \mathbb{E}\left[u_{\varepsilon_{k}}^{+}\left(X^{\delta}\right)\right]\right| \rightarrow 0 .
$$

$c_{0}$ is monotone with respect to $\succeq_{1}$. Hence, Lemma 3.3 implies $R_{0}^{\varepsilon_{k},+} \geq c_{0}\left(R_{\sigma_{k}^{+}}^{\varepsilon_{k},+}\right) \geq c_{0}\left(X^{\delta}\right)$, from which we deduce

$$
\left(u_{\varepsilon_{k}}^{+}\right)^{-1} \circ \mathbb{E}\left[u_{\varepsilon_{k}}^{+}\left(X^{\delta}\right)\right] \geq c_{0}\left(X^{\delta}\right)-\xi_{k} .
$$

Since $X \geq X^{\delta}, X^{\delta}$ assumes only two values, and $c_{0}\left(X^{\delta}\right)$ is a continuity point of $u$, we derive

$$
\mathbb{E}[u(X)] \geq \mathbb{E}\left[u\left(X^{\delta}\right)\right] \geq u\left(c_{0}\left(X^{\delta}\right)\right), \quad \text { as } k \rightarrow \infty .
$$

Finally, if we let $\delta$ tending to zero, it follows $\left\|X^{\delta}-X\right\|_{\infty} \rightarrow 0$ and $\left|c_{0}\left(X^{\delta}\right)-c_{0}(X)\right| \rightarrow 0$. Since $c_{0}(X)$ is a continuity point of $u$, we conclude $\mathbb{E}[u(X)] \geq u\left(c_{0}(X)\right)$. Analogously, if we 
approximate $X$ by $X^{\delta}$ from above and bound it by $R^{\varepsilon_{k},-}$ from below, we get $\mathbb{E}[u(X)] \leq$ $u\left(c_{0}(X)\right)$. Together, we conclude $\mathbb{E}[u(X)]=u\left(c_{0}(X)\right)$ for any random variable $X$ taking at most two values in $(A, B)$ and $c_{0}(X)$ is a continuity point of $u$.

Step 3. The function $u:[A, B] \rightarrow[0,1]$ is continuous. Indeed, suppose by way of contradiction that there is $x \in(A, B]$ such that $u(x-)<u(x)$ (the case $u(x)<u(x+)$ works analogously). Since $c_{0}$ is $\|\cdot\|_{\infty}$-continuous and strictly increasing, there exist sequences $\left(x_{1}^{n}\right)$ and $\left(x_{2}^{n}\right)$ in $(A, B)$ such that

(i) $x_{1}^{n}<x_{2}^{n}<x, x_{1}^{n} \nearrow x_{1}$ and $x_{2}^{n} \nearrow x_{2}=x$,

(ii) $c_{0}\left(X^{n}\right)$ and $c_{0}(X)$ are continuity points of $u$, and

(iii) $\left\|X^{n}-X\right\|_{\infty} \rightarrow 0$,

where $X$ and $X^{n}$ assume the values $\left\{x_{1}, x_{2}\right\}$ and $\left\{x_{1}^{n}, x_{2}^{n}\right\}$ with probabilities $1 / 2$. Indeed, the function $x_{1} \mapsto c_{0}(X)$ maps from $\left(A, x_{2}\right)$ in $\left(A, x_{2}\right)$. Since the set of discontinuity points of $u$ in $\left(A, x_{2}\right)$ is countable and $c_{0}$ is strictly increasing, there are at most countably many $x_{1} \in\left(A, x_{2}\right)$ for which $c_{0}(X)$ is a discontinuity point of $u$. Hence, there is $x_{1} \in\left(A, x_{2}\right)$ for which $c_{0}(X)$ is a continuity point of $u$. We then approximate $X$ by $X^{n}$ from below in $L^{\infty}(A, B)$ such that $c_{0}\left(X^{n}\right)$ are continuity points of $u$. Then, $c_{0}\left(X^{n}\right) \nearrow c_{0}(X)$ and Step 2 yields

$$
\mathbb{E}\left[u\left(X^{n}\right)\right]=u\left(c_{0}\left(X^{n}\right)\right) \nearrow u\left(c_{0}(X)\right)=\mathbb{E}[u(X)],
$$

in contradiction to $u\left(x_{2}^{n}\right) \nearrow u\left(x_{2}-\right)<u\left(x_{2}\right)$. Whence, $u$ has to be continuous. In particular, Step 2 yields $\mathbb{E}[u(X)]=u\left(c_{0}(X)\right)$ for all random variables $X$ taking at most two values in $(A, B)$.

Step 4. The function $u:[A, B] \rightarrow[0,1]$ is strictly increasing. Indeed, by way of contradiction assume there exist $A<r<s<B$ such that $u(r)=u(s)$. Since $u$ is not constant, there exists $t<r$ such that $u(t)<u(r)$ or $t>s$ such that $u(t)>u(s)$. In the second case, denote $v:=\inf \{x>s \mid u(x)>u(s)\}$. By continuity of $u$ one has $s \leq v<t$ and $u(r)=u(s)=u(v)$. Since $c_{0}$ is $\|\cdot\|_{\infty}$-continuous, there exists $w \in(v, t]$ such that $c_{0}(X) \leq v$, where $X$ is a random variable taking the values $r$ and $w$ with probability $1 / 2$ each. If $Y$ is a random variable that is equal to $v$ and $w$ with probabilities $1 / 2$, then $c_{0}(Y)>v$ and therefore, $u\left(c_{0}(Y)\right)>u\left(c_{0}(X)\right)$. On the other hand, $u\left(c_{0}(Y)\right)=\mathbb{E}[u(X)]=$ $\mathbb{E}[u(Y)]=u\left(c_{0}(X)\right)$, which is a contradiction. The proof is completed.

Lemma 2.5 specializes to the present context as follows.

Lemma 3.6 Let $N \in \mathbb{N}$ and $X \in L^{\infty}(A, B)$ be a random variable taking $2^{N}$ different values with strictly positive probabilities. There exists a subsequence of $\left(\varepsilon_{k}\right)$ (still denoted by $\left(\varepsilon_{k}\right)$ ), such that for any $k \in \mathbb{N}$ large enough, we may find stochastic processes $R^{\varepsilon_{k},+}, R^{\varepsilon_{k},-}$ of the form (46) as well as bounded stopping times $\sigma_{k}^{+}$and $\sigma_{k}^{-}$, such that $X$ satisfies

$$
R_{\sigma_{k}^{+}}^{\varepsilon_{k},+} \succeq_{1} X \succeq_{1} R_{\sigma_{k}^{-}}^{\varepsilon_{k},-}
$$

and

$$
\lim _{k \rightarrow \infty}\left|u_{\varepsilon_{k}}^{+}\left(R_{0}^{\varepsilon_{k},+}\right)-\mathbb{E}\left[u_{\varepsilon_{k}}^{+}(X)\right]\right|=\lim _{k \rightarrow \infty}\left|u_{\varepsilon_{k}}^{-}\left(R_{0}^{\varepsilon_{k},-}\right)-\mathbb{E}\left[u_{\varepsilon_{k}}^{-}(X)\right]\right|=0 .
$$


Sketch of the proof. The proof is a straightforward modification of the proof of Lemma 2.5. In a first step, we define $\hat{Y}_{N}^{\varepsilon_{k}}=\hat{X}$ and

$$
\hat{Y}_{n}^{\varepsilon_{k}}=\left(u_{\varepsilon_{k}}^{+}\right)^{-1} \circ \hat{\mathbb{E}}\left[u_{\varepsilon_{k}}^{+}\left(\hat{Y}_{n+1}^{\varepsilon_{k}}+\varepsilon_{k}\right) \mid \hat{\mathcal{F}}_{n}\right], \quad n=0, \ldots, N-1,
$$

on the filtered probability space $\left(\hat{\Omega}, \hat{\mathcal{F}},\left(\hat{\mathcal{F}}_{n}\right)_{n=0}^{N}, \hat{\mathbb{P}}\right)$. Here we assume that $\varepsilon_{k}$ is small enough such that $\hat{Y}_{n}^{\varepsilon_{k}} \in\left(A+\varepsilon_{k}, B-\varepsilon_{k}\right)$ for all $n=0, \ldots, N$. We then define $R^{\varepsilon_{k},+}$ as the stochastic process which follows the dynamics (46) and starts at

$$
R_{0}^{\varepsilon_{k},+}=\min \left\{x \in \mathcal{I}_{\varepsilon_{k}} \mid x \geq \hat{Y}_{0}^{\varepsilon_{k}}\right\} \in(A, B)
$$

The martingale stopping arguments given in the proof of Lemma 2.5 imply the existence of a bounded stopping time $\sigma_{k}^{+}$such that

$$
R_{\sigma_{k}^{+}}^{\varepsilon_{k},+} \succeq_{1} X
$$

Finally, there is a subsequence of $\left(\varepsilon_{k}\right)$, which we still denote by $\left(\varepsilon_{k}\right)$, such that $\hat{Y}_{n}^{\varepsilon_{k}}$ converges to $\hat{Y}_{n}$ for all $n=0, \ldots, N-1$. By continuity of $u$, the arguments given in Step 3 of the proof of Lemma 2.5 imply $\left|u_{\varepsilon_{k}}^{+}\left(\hat{Y}_{0}^{\varepsilon_{k}}\right)-\mathbb{E}\left[u_{\varepsilon_{k}}^{+}(X)\right]\right| \rightarrow 0$. Since $\left|\hat{Y}_{0}^{\varepsilon_{k}}-R_{0}^{\varepsilon_{k},+}\right| \leq \varepsilon_{k}$, we conclude (54). The proof for the lower bound works analogously.

Proof of the "only if"-part of Theorem 1.4.

Let $[A, B]$ denote a compact interval in $(a, b)$. Fix $X \in L^{\infty}(A, B)$ and $\delta>0$. By $\|\cdot\|_{\infty^{-}}$ continuity of $c_{0}$, there exists $X^{\delta}$ taking $2^{N}$ different values, such that $X^{\delta} \in L^{\infty}(A, B)$, $\left\|X^{\delta}-X\right\|_{\infty} \leq \delta$ and $\left|c_{0}\left(X^{\delta}\right)-c_{0}(X)\right| \leq \delta$. Since $c_{0}$ is monotone with respect to $\succeq_{1}$ we deduce from Lemma 3.3 and Lemma 3.6 that

$$
R_{0}^{\varepsilon_{k},+} \geq c_{0}\left(R_{\sigma_{k}^{+}}^{\varepsilon_{k},+}\right) \geq c_{0}\left(X^{\delta}\right) \geq c_{0}\left(R_{\sigma_{k}^{-}}^{\varepsilon_{k},-}\right) \geq R_{0}^{\varepsilon_{k},-}
$$

and therefore

$$
\begin{aligned}
& u_{\varepsilon_{k}}^{+}\left(R_{0}^{\varepsilon_{k},+}\right)-\mathbb{E}\left[u_{\varepsilon_{k}}^{+}\left(X^{\delta}\right)\right] \geq u_{\varepsilon_{k}}^{+}\left(c_{0}\left(X^{\delta}\right)\right)-\mathbb{E}\left[u_{\varepsilon_{k}}^{+}\left(X^{\delta}\right)\right], \\
& u_{\varepsilon_{k}}^{-}\left(c_{0}\left(X^{\delta}\right)\right)-\mathbb{E}\left[u_{\varepsilon_{k}}^{-}\left(X^{\delta}\right)\right] \geq u_{\varepsilon_{k}}^{-}\left(R_{0}^{\varepsilon_{k},-}\right)-\mathbb{E}\left[u_{\varepsilon_{k}}^{-}\left(X^{\delta}\right)\right] .
\end{aligned}
$$

Letting $k$ tending to infinity yields $u^{-1} \circ \mathbb{E}\left[u\left(X^{\delta}\right)\right] \geq c_{0}\left(X^{\delta}\right)$ and $c_{0}\left(X^{\delta}\right) \geq u^{-1} \circ \mathbb{E}\left[u\left(X^{\delta}\right)\right]$ and therefore $c_{0}\left(X^{\delta}\right)=u^{-1} \circ \mathbb{E}\left[u\left(X^{\delta}\right)\right]$. Letting $\delta$ converging to zero, it follows

$$
c_{0}(X)=u^{-1} \circ \mathbb{E}[u(X)] \quad \text { for all } X \in L^{\infty}(A, B),
$$

as $u$ is uniformly continuous on $[A, B]$.

Hence, for any compact interval $[A, B] \subset(a, b)$ there is $u_{A, B}:[A, B] \rightarrow[0,1]$ strictly increasing and continuous such that $c_{0}(X)=u_{A, B}^{-1} \circ \mathbb{E}\left[u_{A, B}(X)\right]$ for all $X \in L^{\infty}(A, B)$. We next give some standard arguments showing that $u_{A, B}$ on $[A, B]$ is uniquely determined up 
to affine transformations. Suppose there exist two strictly increasing, continuous functions $u, \tilde{u}:[A, B] \rightarrow \mathbb{R}$ such that

$$
c_{0}(X)=u^{-1} \circ \mathbb{E}[u(X)]=\tilde{u}^{-1} \circ \mathbb{E}[\tilde{u}(X)] \quad \text { for all } X \in L^{\infty}(A, B) .
$$

Define the strictly increasing and continuous function $\psi(x)=\tilde{u} \circ u^{-1}(x)$. Then $\tilde{u}(x)=$ $\psi \circ u(x)$ and (56) yields $\tilde{u} \circ u^{-1} \circ \mathbb{E}[u(X)]=\mathbb{E}[\tilde{u}(X)]$. This shows

$$
\psi \circ \mathbb{E}[u(X)]=\mathbb{E}[\psi \circ u(X)] \quad \text { for all } X \in L^{\infty}(A, B),
$$

and consequently $\psi(x)=\alpha x+\beta$ for some $\alpha>0$ and $\beta \in \mathbb{R}$ (approximate $\psi$ uniformly on compacts by polynomials). We therefore can extend $u_{A, B}:[A, B] \rightarrow \mathbb{R}$ with $c_{0}(X)=$ $u_{A, B}^{-1} \circ \mathbb{E}\left[u_{A, B}(X)\right]$ for all $X \in L^{\infty}(A, B)$ to $u_{A^{\prime}, B^{\prime}}:\left[A^{\prime}, B^{\prime}\right] \rightarrow \mathbb{R}$ such that $[A, B] \subset\left[A^{\prime}, B^{\prime}\right]$, $u_{A^{\prime}, B^{\prime}}$ restricted to $[A, B]$ coincides with $u_{A, B}$ and $c_{0}(X)=u_{A^{\prime}, B^{\prime}}^{-1} \circ \mathbb{E}\left[u_{A^{\prime}, B^{\prime}}(X)\right]$ for all $X \in L^{\infty}\left(A^{\prime}, B^{\prime}\right)$. By exhausting $(a, b)$ with compact intervals $\left[A^{n}, B^{n}\right] \subset(a, b)$, there is a continuous and strictly increasing function $u:(a, b) \rightarrow \mathbb{R}$ which is unique up to affine transformations and satisfies $c_{0}(X)=u^{-1} \circ \mathbb{E}[u(X)]$ for all $X \in L^{\infty}(a, b)$ with values in some compact interval $[A, B] \subset(a, b)$. By $\|\cdot\|_{\infty}$-continuity of $c_{0}$ it follows that $c_{0}(X)=$ $u^{-1} \circ \mathbb{E}[u(X)]$ for all $X \in L^{\infty}(a, b)$ and the proof of Theorem 1.4 is completed.

\section{A Helly's theorem}

The following lemma is well-known. For the sake of completeness we give a proof.

Lemma A.1 Let $f_{n}:[A, B] \rightarrow[0,1]$ be a sequence of increasing, continuous functions. Then, there is a subsequence $\left(f_{n_{k}}\right)$ and an increasing function $f:[A, B] \rightarrow[0,1]$ such that

$$
f_{n_{k}}(x) \rightarrow f(x) \quad \text { for all } x \in[A, B] \text { as } k \rightarrow \infty .
$$

The function $f$ has at most countably many discontinuity points. Moreover, $f_{n_{k}}\left(x_{k}\right) \rightarrow f(x)$ for any sequence $x_{k} \in[A, B]$ which converges to some continuity point $x \in[A, B]$ of $f$.

Remark A.2 Helly's theorem is usually stated as a convergence result only for the continuity points of the limiting function $f$. It was observed in Campi and Schachermayer [11] that one may also obtain convergence on the discontinuity points of $f$.

Proof. Let $\left(z_{j}\right)_{j \in \mathbb{N}}$ be a sequence running through $\mathcal{I}:=[A, B] \cap \mathbb{Q}$. Let $\left(f_{n_{k}^{1}}\right)$ be a subsequence of $\left(f_{n}\right)$ such that $f_{n_{k}^{1}}\left(z_{1}\right)$ converges to $f\left(z_{1}\right) \in[0,1]$. Let $\left(f_{n_{k}^{2}}\right)$ be a subsequence of $\left(f_{n_{k}^{1}}\right)$ such that $f_{n_{k}^{2}}\left(z_{2}\right) \rightarrow f\left(z_{2}\right) \in[0,1]$ and so on. Then, $f_{n_{k}^{k}}(x) \rightarrow f(x)$ for all $x \in \mathcal{I}$. The function $f$ is increasing on $\mathcal{I}$, i.e., for any $x, y \in \mathcal{I}$ with $x \leq y$ it follows $f(x) \leq f(y)$. Therefore, $f$ has at most countably many discontinuities $\left(z_{j}\right)_{j \geq 1}$. Let $\left(f_{\xi_{k}}\right)$ be a subsequence of $\left(f_{n_{k}^{k}}\right)$ such that $f_{\xi_{k}^{1}}(x) \rightarrow f(x)$ for all $x \in \mathcal{I} \cup\left\{z_{1}\right\},\left(f_{\xi_{k}^{2}}\right)$ a subsequence of $\left(f_{\xi_{k}^{1}}\right)$ such that $f_{\xi_{k}^{2}}\left(z_{2}\right) \rightarrow f\left(z_{2}\right)$ and so on. Define $f_{n_{k}}:=f_{\xi_{k}^{k}}$ which is a subsequence of $\left(f_{n}\right)$. Then, $f_{n_{k}}(x) \rightarrow f(x)$ for all $x \in \bigcup_{j \geq 1}\left\{z_{j}\right\} \cup \mathcal{I}$. By construction, any $x \in[A, B] \backslash\left(\bigcup_{j \geq 1}\left\{z_{j}\right\}\right)$ is a 
continuity point of $f: \mathcal{I} \rightarrow[0,1]$, whence we define $f(x)=\lim _{n \rightarrow \infty} f\left(x_{n}\right)$ for an arbitrary sequence $x_{n} \in \mathcal{I}$ converging to $x$. The function $f:[A, B] \rightarrow[0,1]$ is increasing.

Let $x_{k} \in[A, B]$ be a sequence with limit $x$, and assume that $f$ is continuous at $x$. Fix $\delta>0$. There exist $y_{1}, y_{2} \in \mathcal{I}$ with $y_{1}<x<y_{2}$ such that $|f(y)-f(x)| \leq \delta / 4$ for all $y \in\left[y_{1}, y_{2}\right]$. Furthermore, there is $k_{0} \in \mathbb{N}$ such that

$$
y_{1} \leq x_{k} \leq y_{2}, \quad\left|f_{n_{k}}\left(y_{1}\right)-f\left(y_{1}\right)\right| \leq \frac{\delta}{4}, \quad\left|f_{n_{k}}\left(y_{2}\right)-f\left(y_{2}\right)\right| \leq \frac{\delta}{4} \quad \text { for all } k \geq k_{0} .
$$

Then

$$
\begin{aligned}
\left|f_{n_{k}}\left(x_{k}\right)-f(x)\right| & \leq\left|f_{n_{k}}\left(y_{2}\right)-f(x)\right|+\left|f(x)-f_{n_{k}}\left(y_{1}\right)\right| \\
& \leq\left|f_{n_{k}}\left(y_{2}\right)-f\left(y_{2}\right)\right|+\left|f\left(y_{1}\right)-f_{n_{k}}\left(y_{1}\right)\right|+\frac{\delta}{2} \leq \delta \quad \text { for all } k \geq k_{0} .
\end{aligned}
$$

In particular, $f_{n_{k}}(x) \rightarrow f(x)$ for all $x \in[A, B]$ and $f$ is increasing. The proof is completed. $\square$

\section{References}

[1] Alt, F. (1935). Über die Messbarkeit des Nutzens. Zeitschrift für Nationalökonomie, Verlag von Julius Springer, Wien.

[2] Artzner, Ph., Delbaen, F., Eber, J.M., Heath, D. (1999). Coherent Risk Measures. Mathematical Finance, 9(3), 203-228.

[3] Artzner, P., Delbaen, F.; Eber, J.-M.; Heath, D., Ku, H. (2007). Coherent multiperiod risk adjusted values and Bellman's principle. Ann. Oper. Res. 152, 5-22.

[4] Bernoulli, D. (1738). Specimen theoriae novae de mensura sortis. Commentarii Academiae Scientarum Imperalis Petropolitanae 5, 175-192. Translated by L. Sommer: Econometrica 22 (1954), 23-36.

[5] Bullen, P.S. (2003). Handbook of Means and Their Inequalities. Kluwer, Dordrecht.

[6] Campi, L., Schachermayer, W. (2006) A Super-Replication Theorem in Kabanov's Model of Transaction Costs. Finance and Stochastics 10(4), 579-596.

[7] Cerreia-Vioglio, S.,Maccheroni, F., Marinacci, M., Montrucchio, L. (2009). Risk Measures: Rationality and Diversification. Preprint.

[8] Cheridito, P., Delbaen, F., Kupper, M. (2006). Dynamic monetary risk measures for bounded discrete-time processes. Electronic J. Probab. 11, 57-106.

[9] Cheridito, P., Kupper, M. (2007). Composition of Time-Consistent Dynamic Monetary Risk Measures in Discrete Time. Preprint.

[10] Cheridito, P., Li, T. (2008). Dual characterization of properties of risk measures on Orlicz hearts. Mathematics and Financial Economics 2(1), 29-55. 
[11] Cheridito, P., Stadje, M. (2009). Time-inconsistency of VaR and time-consistent alternatives. Finance Research Letters, 6(1), 40-46.

[12] Delbaen, F.: Coherent risk measures on general probability spaces. Advances in Finance and Stochastics, 1-37, Springer, Berlin (2002)

[13] Delbaen, F. (2006). The structure of m-stable sets and in particular of the set of riskneutral measures. In: Yor, M., Emery, M. (eds.): In Memoriam Paul-André Meyer - Seminaire de Probabilités XXXIX. Berlin Heidelberg New York: Springer, 215-258.

[14] Delbaen, F., Peng, S., Rosazza Gianin, E. (2008). Representation of the penalty term of dynamic concave utilities. Preprint.

[15] Ellsberg, D. (1961). Risk, ambiguity and Savage axioms. Quart. J. Econom. 75, 643669.

[16] de Finetti, B. (1931). Sul concetto di media. Giornale dell'Instituto Italiano degli Attuari 2, 369-396.

[17] Föllmer, H., Penner, I. (2006). Convex risk measures and the dynamics of their penalty functions. Stat. Decisions 24(1), 61-96.

[18] Föllmer, H., Schied, A., (2002c). Stochastic Finance, An Introduction in Discrete Time. de Gruyter Studies in Mathematics $2 \%$.

[19] Frittelli, M., Rosazza Gianin, E. (2005). Law-invariant convex risk measures. Advances in Mathematical Economics 7, 33-46.

[20] Gilboa, I., Schmeidler, D. (1989). Maxmin expected utility with non-unique prior. J. Math. Econom., 18, 141-153.

[21] Gerber, H.U. (1979). An Introdution to Mathematical Risk Theory. S.S. Huebner Foundation for Insurance Education, Wharton School, University of Pennsylvania, Philadelphia.

[22] Gerber, H.U. (1974). On iterative Premium Calculation Principles. Sonderabdruck aus den Mitteilungen der Vereinigung schweizerischer Versicherungsmathematiker, 74. Band, Heft 2.

[23] Gerber, H.U., Goovaerts, M. (1981). On the representation of additive principles of premium calculation. Scand. Actuarial J., 4, 221-227.

[24] Goovaerts, M., Kaas, R., Laeven, R., Tang, Q. (2004). A comonotonic image of independence for additive risk measures. Insurance Mathematics and Economics, 35, $581-594$.

[25] Hardy, G., Littlewood, J.E., Pólya, G. (1952). Inequalities. 2nd ed., Cambridge University press, Cambridge. 
[26] Jouini, E., Schachermayer, W., Touzi, N. (2006). Law invariant risk measures have the Fatou property. Advances in Mathematical Economics, 9, 49-71, Springer, Tokyo.

[27] Kolmogorov, A.N. (1930). Sur la notion de la moyenne. Atti della R. Accademia Nazionale dei Lincei, 12, 388-391.

[28] Kusuoka S. (2001). On law-invariant coherent risk measures. Advances in Mathematical Economics, 3, 83-95.

[29] Machina, M. (1989). Dynamic Consistency and Non-Expected Utility Models of Choice Under Uncertainty. Journal of Economic Literature, 27, 1622-1668.

[30] Maccheroni, F., Marinacci, M., Rustichini, A. (2006). Ambiguity aversion, robustness, and the variational representation of preferences. Econometrica 74, 1447-1498.

[31] Nagumo, M. (1930). Über eine Klasse der Mittelwerte. Japanese Journal of Mathematics 7, 71-79.

[32] Von Neumann, J., Morgenstern, O. (1944). Theory of Games and Economic Behavior. Princeton University Press, Princeton, New Jersey.

[33] Revuz, D., Yor, M., (1994). Continuous Martingales and Brownian Motion. Second edition, Springer-Verlag, Berlin.

[34] Savage, L. J. (1954). The foundations of statistics. Wiley Publ. Stat., John Wiley and Sons, New York.

[35] Weber, S. (2006). Distribution-Invariant Risk Measures, Information, and Dynamic Consistency. Mathematical Finance, 16(2), 419-442. 